\title{
Optimizing Main-Memory Join on Modern Hardware
}

\author{
Stefan Manegold, Peter Boncz, Member, IEEE, and Martin Kersten, Member, IEEE Computer Society
}

\begin{abstract}
In the past decade, the exponential growth in commodity CPU's speed has far outpaced advances in memory latency. A second trend is that CPU performance advances are not only brought by increased clock rate, but also by increasing parallelism inside the CPU. Current database systems have not yet adapted to these trends and show poor utilization of both CPU and memory resources on current hardware. In this paper, we show how these resources can be optimized for large joins and translate these insights into guidelines for future database architectures, encompassing data structures, algorithms, cost modeling, and implementation. In particular, we discuss how vertically fragmented data structures optimize cache performance on sequential data access. On the algorithmic side, we refine the partitioned hash-join with a new partitioning algorithm called radix-cluster, which is specifically designed to optimize memory access. The performance of this algorithm is quantified using a detailed analytical model that incorporates memory access costs in terms of a limited number of parameters, such as cache sizes and miss penalties. We also present a calibration tool that extracts such parameters automatically from any computer hardware. The accuracy of our models is proven by exhaustive experiments conducted with the Monet database system on three different hardware platforms. Finally, we investigate the effect of implementation techniques that optimize CPU resource usage. Our experiments show that large joins can be accelerated almost an order of magnitude on modern RISC hardware when both memory and CPU resources are optimized.
\end{abstract}

Index Terms-Main-memory databases, query processing, memory access optimization, decomposed storage model, join algorithms, implementation techniques.

\section{INTRODUCTION}

C USTOM hardware-from workstations to PCs-has experienced tremendous performance improvements in the past decades. Unfortunately, these improvements are not equally distributed over all aspects of hardware performance and capacity. Fig. 1 shows that the speed of commercial microprocessors has increased roughly 70 percent every year, while the speed of commodity DRAM has improved by little more than 50 percent over the past decade [1]. One reason for this is that there is a direct tradeoff between capacity and speed in DRAM chips, and the highest priority has been for increasing capacity. The result is that, from the perspective of the processor, memory is getting slower at a dramatic rate, making it increasingly difficult to achieve high processor efficiencies. Another trend is the ever-increasing number of interstage and intrastage parallel execution opportunities provided by multiple execution pipelines and speculative execution in modern CPUs. Current database systems on the market make poor use of these new features; studies on several DBMS products on a variety of workloads [2], [3], [4], [5] consistently show that modern CPUs are stalled (i.e., nonworking) most of the execution time.

In this paper, we show how large main-memory joins can be accelerated by optimizing memory and CPU resource utilization on modern hardware. These optimizations involve radical changes in database architecture,

- The authors are with CWI, Kruislaan 413, 1098 SJ Amsterdam, The Netherlands. E-mail: \{manegold, boncz, mk\}@cwi.nl.

Manuscript received 8 Oct. 1999; revised 10 Nov. 2000; accepted 14 Dec. 2000; posted to Digital Library 7 Sept. 2001.

For information on obtaining reprints of this article, please send e-mail to: tkde@computer.org, and reference IEEECS Log Number 110731. encompassing new data structures, query processing algorithms, and implementation techniques. Our findings are summarized as follows:

- Memory access is a bottleneck to query processing. We demonstrate that the performance of even simple database operations is severely constrained by memory access costs. For example, a simple inmemory table scan runs on Sun hardware from the year 2000 in roughly the same absolute time as on a Sun from 1992, now spending 95 percent of its cycles waiting for memory (see Section 2.2). It is important to note that this bottleneck affects database performance in general, not only main-memory database systems.

- Data structures and algorithms should be tuned for memory access. We discuss database techniques to avoid the memory access bottleneck, both in the fields of data structures and query processing algorithms. The key issue is to optimize the use of the various caches of the memory subsystem. We show how vertical table fragmentation optimizes sequential memory access to column data. For equi-join, which has a random access pattern, we refine partitioned hash-join with a new radix-cluster algorithm which makes its memory access pattern more easy to cache. Our experiments indicate that large joins can strongly benefit from these techniques.

- Memory access costs can be modeled precisely. Cacheaware algorithms and data structures must be tuned to the memory access pattern imposed by a query and hardware characteristics such as cache sizes and miss penalties, just like traditional query 


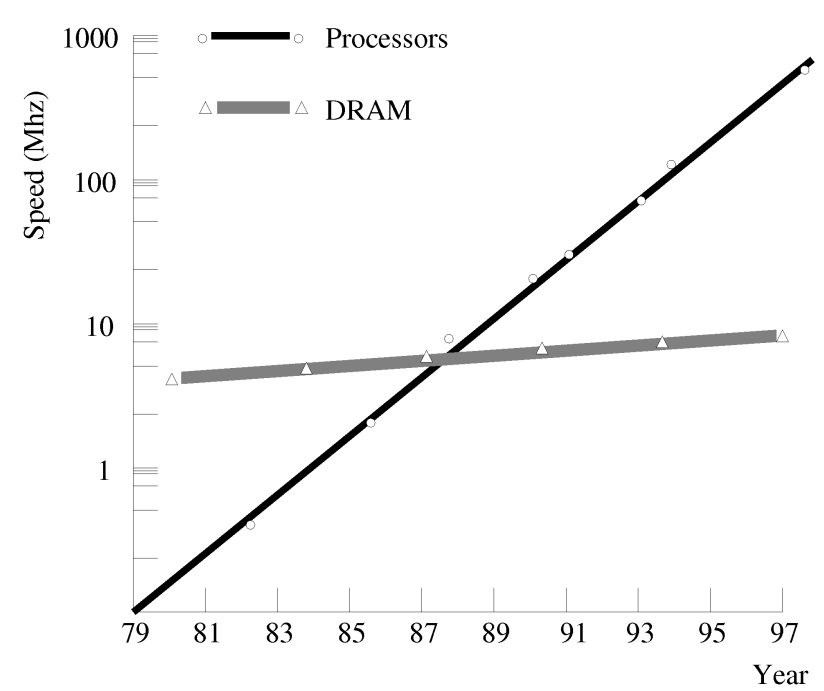

Fig. 1. Trends in DRAM and CPU speed.

optimization tunes the I/O pattern imposed by a query to the size of the buffers available and $\mathrm{I} / \mathrm{O}$ cost parameters. Therefore, it is necessary to have models that predict memory access costs in detail. In this work, we provide such detailed models for our partitioned hash-join algorithms. These models use an analytical framework that predicts the number of hardware events (e.g., cache misses and CPU cycles) and scores them with hardware parameters. We also outline our calibration tool which extracts these cost parameters automatically from any computer system.

- Memory optimization and efficient coding techniques boost each others effects. CPU resource utilization can be optimized with implementation techniques known from high-performance computing [6] and main-memory database systems [7], [8]. We observe that applying these optimizations in combination with memory optimizations yields a higher performance increase than applying them without memory optimizations. The same is also the case for memory optimizations: They turn out to be more effective on CPU-optimized code than on nonoptimized code. Our experiments show that database performance can be improved by an order of magnitude by applying both CPU and memory optimization techniques.

Our research group has studied large main-memory database systems for the past 10 years. This research started in the PRISMA project [9], focusing on massive parallelism, and is now centered around Monet [10], [11]: a highperformance system targeted to query-intensive application areas like OLAP and data mining. We use Monet as our experimentation platform.

\subsection{Related Work}

Database system research into the design of algorithms and data structures that optimize memory access has been relatively scarce. Our major reference is the work by Shatdal et al. [12], which shows that join performance can be improved using a main-memory variant of Grace Join in which both relations are first hash-partitioned in chunks that fit the (L2) memory cache. There were various reasons that led us to explore this direction of research further. First, after its publication, the observed trends in custom hardware have continued, deepening the memory access bottleneck. For instance, the authors list a mean performance penalty for a cache miss of 20-30 cycles in 1994, while a range of 50-100 is typical in 2000 (and rising). This increases the benefits of cache optimizations and possibly changes the trade-offs. Another development has been the introduction of so-called level-one (L1) caches, which are typically very small regions on the CPU chip that can be accessed at almost CPU clockspeed. The authors of [12] provide algorithms that are only feasible for the relatively larger off-chip L2 caches. Finally, this previous work uses standard relational data structures, while we argue that the impact of memory access is so severe that vertically fragmented data structures should be applied at the physical level of database storage.

Though we consider memory-access optimization to be relevant for database performance in general, it is especially important for main-memory databases, a field that, through time, has received fluctuating interest within the database research community. In the 1980s [13], [14], [15], [16], [17], [18], falling DRAM prices seemed to suggest that most data would soon be memory-resident; its popularity diminished in the 1990s, narrowing its field of application to real-time systems only. Currently, interest has revived into applications for small and distributed database systems, but also in high-performance systems for query-intensive applications like data mining and OLAP. In our research, we focus on this latter category. Example commercial systems are the Times Ten product [19], Sybase IQ [20], and Compaq's Infocharger [21], which is based on an early version of Monet [8], developed by our own group since 1994 and commercially deployed in a data mining tool [22]. Monet is implemented using aggressive coding techniques for optimizing CPU resource utilization [8] that go much beyond the usual MMDBS implementation techniques [23]. For example, Monet is written in a macrolanguage from which $C$ language implementations are generated. The macros implement a variety of techniques by virtue of which the inner loops of performance-critical algorithms like join are free of overheads like database ADT calls, data movement, and loop condition management. These techniques were either pioneered by our group (e.g., logarithmic code expansion [7]) or taken from the field of high-performance computing [6]. In this work, we will show that these techniques allow compilers to produce code that better exploits the parallel resources offered by modern CPUs.

Past work on main-memory query optimization [24], [25] models the main-memory costs of query processing operators on the coarse level of procedure calls, using profiling to obtain some "magical" constants. As such, these models do not provide insight into individual components that make up query costs, which limits their predictive value. Conventional (i.e., non-main-memory) cost modeling, in contrast, has I/O as the dominant cost aspect, which makes it possible to formulate accurate models based on the amount of predicted I/O work. Calibrating such models is relatively easy as statistics on the $\mathrm{I} / \mathrm{O}$ accesses caused 
during an experiment are readily available in a database system. Past work on main-memory systems was unable to provide such cost models on a similarly detailed level for two reasons. First, it was difficult to model the interaction between low-level hardware components like CPU, Memory Management Unit, bus, and memory caches. Second, it was impossible to measure the status of these components during experiments, which is necessary for tuning and calibration of models. Modern CPUs, however, contain performance counters for events like cache misses and exact CPU cycles [26], [27], [28]. This enabled us to develop a new main-memory cost modeling methodology that first mimics the memory access pattern of an algorithm, yielding a number of CPU cycle and memory cache events, and then scores this pattern with an exact cost prediction. Therefore, the contribution of the algorithms, models, and experiments presented here is to demonstrate that detailed cost modeling of main-memory performance is both important and feasible.

\subsection{Outline}

In Section 2, we describe the aspects of memory and CPU technology found in custom hardware that are most relevant for the performance of main-memory query execution. We identify ongoing trends and outline their consequences for database architecture. In addition, we describe our calibration tool, which extracts the most important hardware characteristics like cache size, cache line size, and cache latency from any computer system, and provide results for our benchmark platforms (modern SGI, Sun, Intel, and AMD hardware).

In Section 3, we introduce the radix-cluster algorithm, which improves the partitioning phase in partitioned hashjoin by trading memory access costs for extra CPU processing. We perform exhaustive experiments where we use CPU event counters to obtain detailed insight into the performance of this algorithm. First, we vary the partition sizes to show the effect of tuning the memory access pattern to the memory cache sizes. Second, we investigate the impact of code optimization techniques for main-memory databases. These experiments show that improvements of almost an order of magnitude can be obtained by combining both techniques (cache tuning and code optimization) rather than by each one individually. Our results are fully explained by detailed models of both the partition (radixcluster) and join phase of partitioned hash-join and we show how performance can be exactly predicted from hardware events like cache and TLB misses.

In Section 4, we evaluate our findings and show how they support the choices we made back in 1994 when designing Monet, which uses full vertical fragmentation and implementation techniques optimized for main memory to achieve high performance on modern hardware. We conclude with recommendations for future systems.

\section{Modern Hardware and DBMS Performance}

First, we describe the technical details of modern hardware relevant for main-memory query performance, focusing on CPU and memory architectures. We perform experiments to illustrate how the balance between CPU and memory costs in query processing has shifted through time and discuss a calibration tool that automatically extracts the

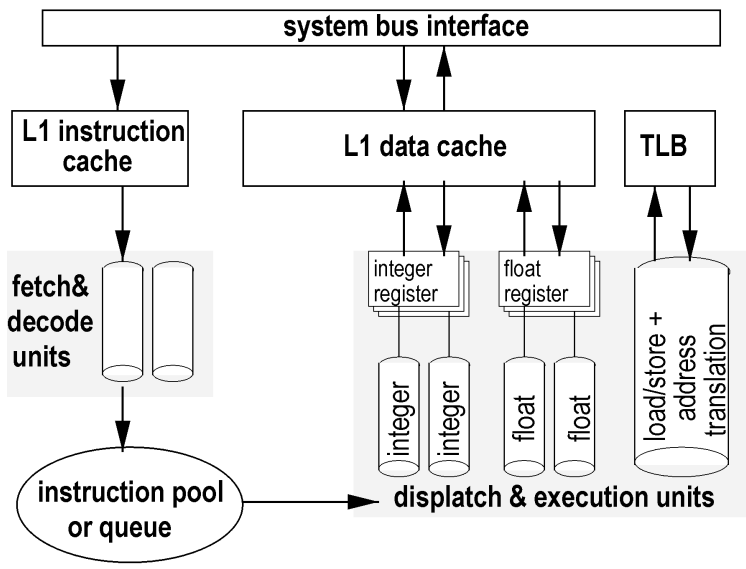

Fig. 2. Modern out-of-order CPU.

hardware parameters most important for performance prediction from any computer system. We then look at what future hardware technology has in store and identify a number of trends.

\subsection{A Short Hardware Primer}

While CPU clock frequency has been following Moore's law (doubling every 18 months), CPUs have additionally become faster through parallelism within the processor. Scalar CPU's separate different execution stages for instructions, e.g., allowing a computation stage of one instruction to be overlapped with the decoding stage of the next instruction. Such a pipelined design allows for interstage parallelism. Modern superscalar CPUs add intrastage parallelism as they have multiple copies of certain (pipelined) units that can be active simultaneously. Although CPUs are commonly classified as either RISC or CISC, modern CPUs combine successful features of both. Fig. 2 shows a simplified schema that characterizes how modern CPUs work: Instructions that need to be executed are loaded from memory by a fetch-and-decode unit. In order to speed up this process, multiple fetch-and-decode units may be present (e.g., the Pentium III and the Athlon have three, the R10000 two). Decoded instructions are placed in an instruction queue from which they are executed by one of various functional units which are sometimes specialized in integer, floating-point, and load/ store pipelines. The Pentium III, for instance, has two such functional units, the R10000 has five, and the Athlon has nine. To exploit this parallel potential, modern CPUs rely on techniques like branch prediction to predict which instruction will be next before the previous has finished. Also, modern cache memories are nonblocking, which means that a cache miss does not stall the CPU. Such a design allows the pipelines to be filled with multiple instructions that will probably have to be executed (also known as speculative execution), betting on yet unknown outcomes of previous instructions. All this is accompanied by the necessary logic to restore order in case of mispredicted branches. As this can cost a significant penalty, and as it is very important to fill all pipelines to obtain the performance potential of the CPU, much attention is paid in hardware design to efficient 


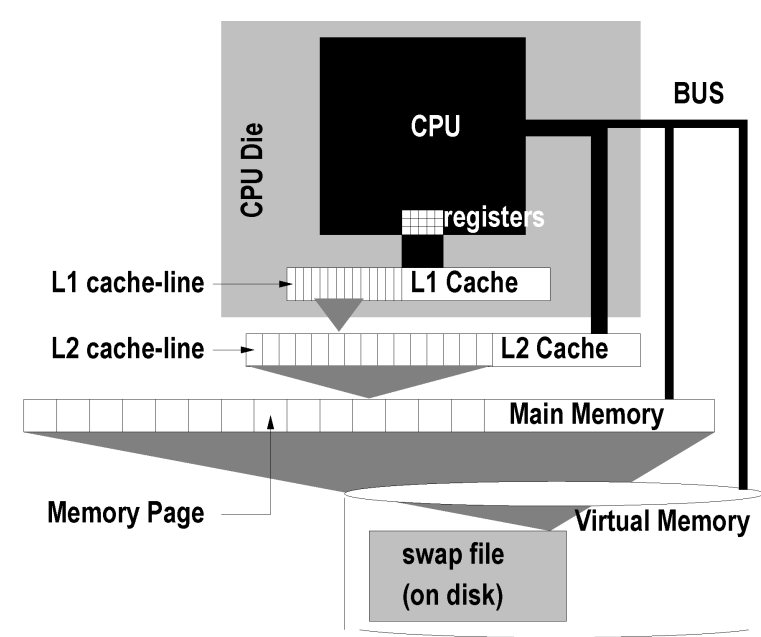

Fig. 3. Hierarchical memory system.

branch prediction. CPUs work with prediction tables that record statistics about branches taken in the past.

Modern computer architectures have a hierarchical memory system, as depicted in Fig. 3, where access by the CPU to main memory, consisting of DRAM chips on the system board, is accelerated by various levels of cache memories. Introduction of these cache memories, which consist of fast but expensive SRAM chips, was necessary due to the fact that DRAM memory latency has progressed little through time, making its performance relative to the CPU become worse exponentially. First, one level of cache was added by placing SRAM chips on the motherboard. Then, as CPU clock-speeds kept increasing, the physical distance between these chips and the CPU became a problem as it takes a minimum amount of time per distance to carry an electrical signal over a wire. As a result, modern CPUs have cache memories inside the processor chip. For simplicity of presentation, we assume one on-chip cache, called L1, and a typically larger off-chip cache on the system board, called L2. Our results, however, also hold for more complex configurations, e.g., with the L2 cache included on the CPU chip and an optional off-chip L3 cache. We identify three aspects that determine memory access costs:

Latency. Our exact definition of memory latency $\left(l_{\mathrm{Mem}}\right)$ is the time needed to transfer one byte from the main memory to the L2 cache. This occurs when the piece of memory being accessed is in neither the L1 nor the L2 cache, so we speak of an L2 miss. It is important to note that, during this time, all current hardware actually transfers multiple consecutive words to the memory subsystem since each cache level has a smallest unit of transfer (called the cache line). During one memory fetch, modern hardware loads an entire cache line from main memory ${ }^{1}$ in one go by reading data from many DRAM chips at the same time, transferring all bits in the cache line in parallel over a wide bus. Similarly, with L2 latency $\left(l_{L 2}\right)$, we mean the time it takes the CPU to access data that is

1. To which cache line the memory is loaded is determined from the memory address. An X-way associative cache allows us to load a line in X different positions. If $\mathrm{X}>1$, some cache replacement policy chooses one from the $X$ candidates. Least Recently Used (LRU) is the most common replacement algorithm. in L2, but not in L1 (an L1 miss), and L1 latency $\left(l_{L 1}\right)$ is the time it takes the CPU to access data in L1. Each L2 miss is preceded by an L1 miss. Hence, the total latency to access data that is in neither cache is $l_{M e m}+l_{L 2}+l_{L 1}$. As L1 latency cannot be avoided, we assume in the remainder of this paper that L1 latency is included in the pure CPU costs and regard only memory latency and L2 latency as explicit memory access costs.

Bandwidth. We define memory bandwidth as the number of megabytes of main memory the CPU can access per second. On some architectures, there is a difference between read and write bandwidth, but this difference tends to be small. Bandwidth is usually maximized on a sequential access pattern as only then are all memory words in the cache lines fully used. In conventional hardware, the memory bandwidth used is simply the cache line size divided by the memory latency, but modern multiprocessor systems typically provide excess bandwidth capacity.

Address translation. The Translation Lookaside Buffer (TLB) is a common element in modern CPUs (see Fig. 2). This buffer is used in the translation of logical virtual memory addresses used by application code to physical page addresses in the main memory of the computer. The TLB is a kind of cache that holds the translation for the most recently used pages (typically 64). If a logical address is found in the TLB, the translation has no additional costs. However, if a logical address is not cached in the TLB, a TLB miss occurs. The more pages an application uses (which is also dependent on the often configurable size of the memory pages), the higher the probability of TLB misses. A TLB miss can either be handled in hardware or in software, depending on the computer architecture. Hardware-handled TLB fetches the translation from a fixed memory structure, which is just filled by the operating system. Softwarehandled TLB leaves the translation method entirely to the operating system, but requires trapping to a routine in the operating system kernel on each TLB miss. Depending on the implementation and hardware architecture, TLB misses can therefore be more even costly than a main-memory access. Moreover, as address translation often requires accessing some memory structure, this can in turn trigger additional memory cache misses.

\subsection{Experimental Quantification}

We use a simple scan test to demonstrate the severe impact of memory access costs on the performance of elementary database operations. In this test, we sequentially scan an inmemory buffer by iteratively reading one byte with a varying stride, i.e., the offset between two subsequently accessed memory addresses. We make sure that the buffer is in memory, but not in any of the memory caches, by first scanning it and then scanning some other buffer larger than the largest cache size multiple times. Our experiment mimics what happens if a database server performs a read-only scan of a one-byte column in an in-memory table with a certain record-width (the stride) as would happen in 


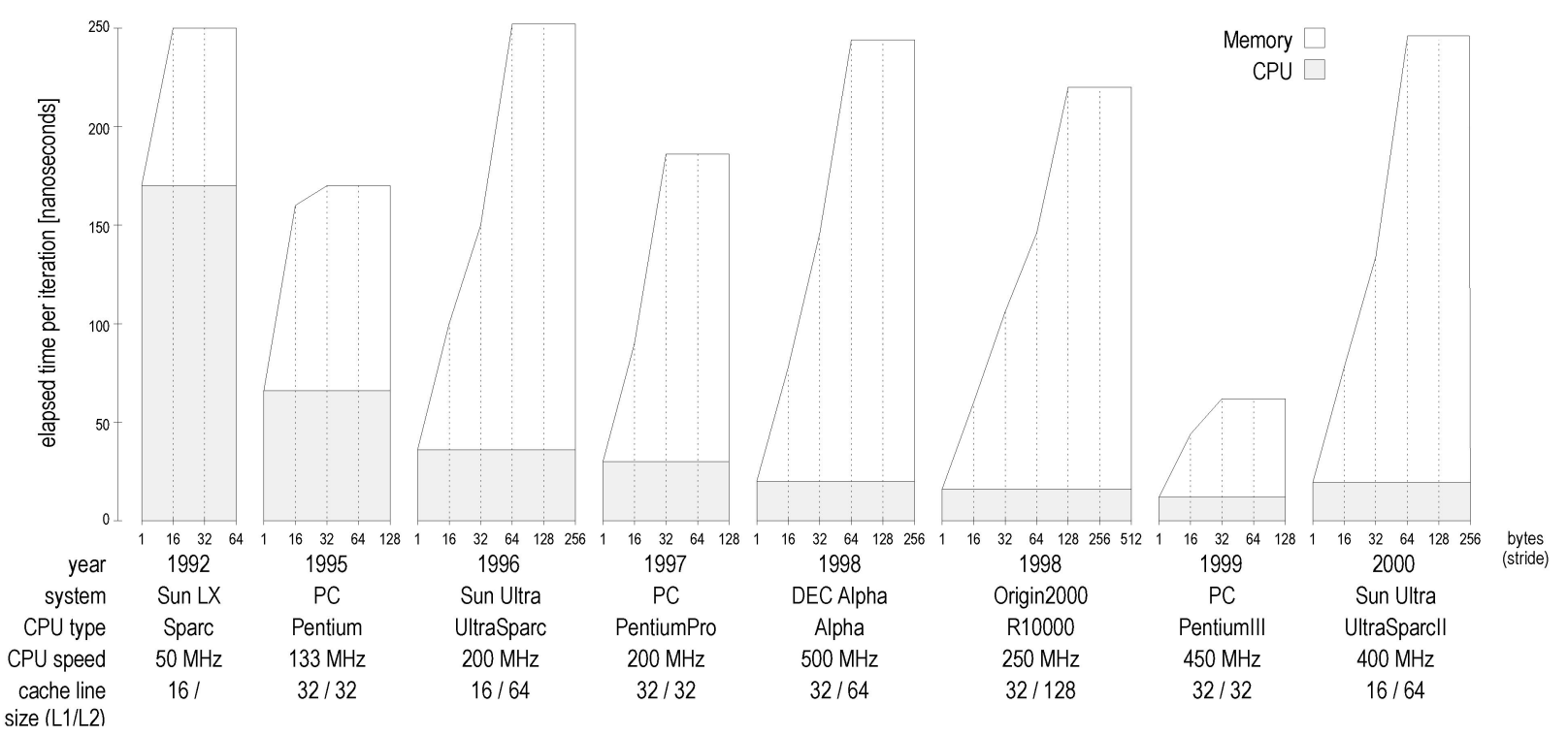

Fig. 4. CPU and memory access costs per tuple in a simple table scan.

a simple aggregation (e.g., SELECT MAX(column) FROM table).

Fig. 4 shows results of this experiment on a number of popular workstations of the past decade. The X-axis shows the different systems, ordered by their age and, per system, the different strides tested. The Y-axis shows the absolute elapsed time for the experiments. For each system, the graph is split up to show which part of the elapsed time is spent waiting for memory (upper) and which part for CPU processing (lower, gray-shaded).

All systems show the same basic behavior, with best performance at stride 1 , increasing to some maximum at a larger stride, after which performance stays constant. This is explained as follows: When the stride is small, successive iterations in the scan read bytes that are near to each other in memory and hit the same cache line. Therefore, the number of L1 and L2 cache misses is low and the memory access costs are negligible compared to the CPU costs. As the stride increases, the cache miss rates and, thus, the memory access costs also increase. The cache miss rates reach their maxima as soon as the stride reaches the cache line size. Then, every memory read is a cache miss. Performance cannot become any worse and stays constant.

When comparing the Sun LX to the Origin2000, we see that CPU performance has increased 10-fold, of which a factor 5 can be attributed to faster clock frequency (from 50 $\mathrm{MHz}$ to $250 \mathrm{MHz}$ ), and a factor 2 to increased processor parallelism (the CPU costs have fallen from 160 ns at 50 $\mathrm{MHz}=8$ cycles to $16 \mathrm{~ns}$ at $250 \mathrm{MHz}=4$ cycles). While this trend of exponentially increasing CPU performance is easily recognizable, the memory cost trend in Fig. 4 shows a mixed picture and has clearly not kept up with the advances in CPU power. Consequently, while our experiment was still largely CPU-bound on the Sun from 1992, it is dominated by memory access costs on the modern machines (even the Pentium III with fast memory spends 75 percent of the time waiting for memory). Note that the later machines from Sun, Silicon Graphics, and DEC actually have memory access costs that, in absolute numbers, are even higher than on the Sun from 1992. This can be attributed to the complex memory subsystem that comes with SMP architectures, resulting in a high memory latency. These machines do provide a high memory bandwidth - thanks to the ever-growing cache line sizes ${ }^{2}$ - but this does not do any good in our experiment at large strides (when data locality is low).

This simple experiment also makes it clear why database systems are quickly constrained by memory access, even on simple tasks like scanning that seem to have an access pattern that is easy to cache (sequential). The default physical representation of a tuple is a consecutive byte sequence (a "record") which must always be accessed by the bottom operators in a query evaluation tree (typically, selections or projections). The record byte-width of typical relational table amounts to some hundreds of bytes. Fig. 4 makes it clear that such large strides lead to worst-case performance such that the memory access bottleneck kills all CPU performance advances.

To improve performance, we strongly recommend using vertically fragmented data structures. In Monet, we fully decompose relational tables on all columns, storing each in a separate Binary Association Tables (BAT). This approach is known in the literature as the Decomposed Storage Model [29]. A BAT is represented in memory as an array of fixed-size two-field records [OID, value]—called Binary UNits (BUN) - where the OIDs are used to link together the tuples that are decomposed across different BATs. Full vertical fragmentation keeps the database records thin ( 8 bytes or less) and is therefore the key for reducing memory access costs (staying on the left side of the graphs in Fig. 4). In Section 4, we will come back to specific implementation details of Monet.

2. In one cache miss, the Origin 2000 fetches 128 bytes, whereas the Sun LX fetches only 16, an improvement of factor 8 . 


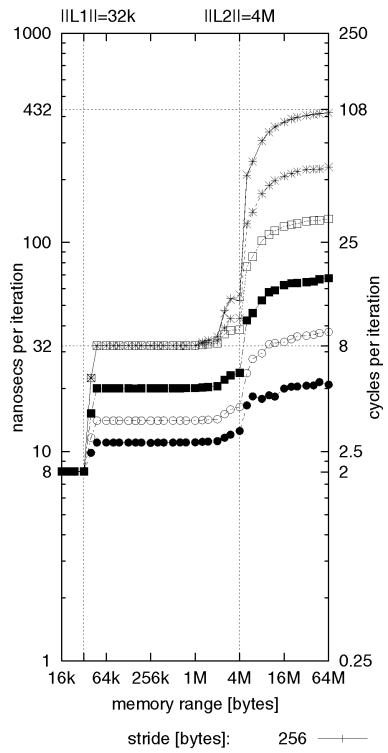

(a)

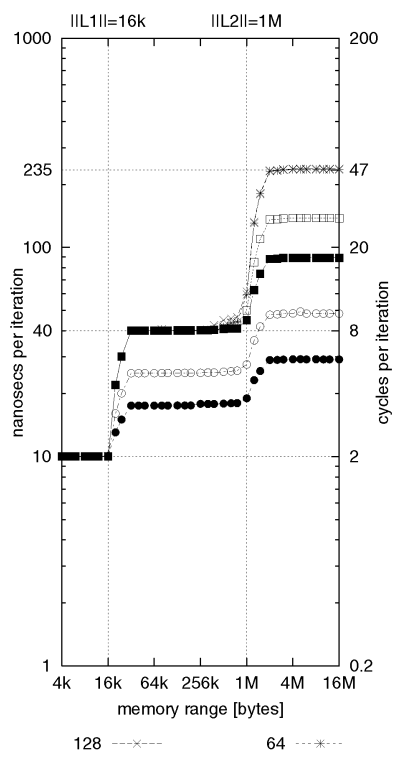

(b)

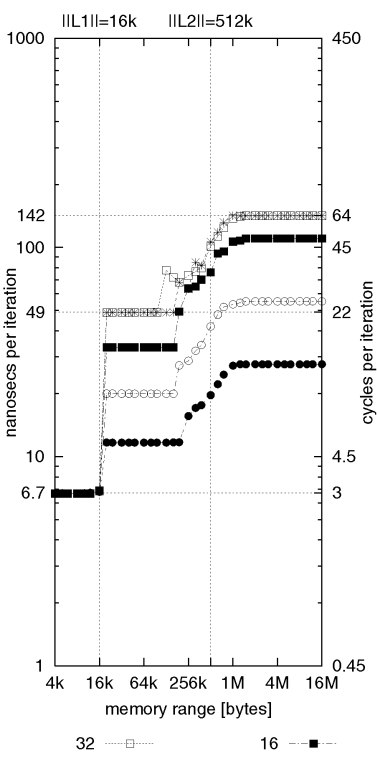

(c)

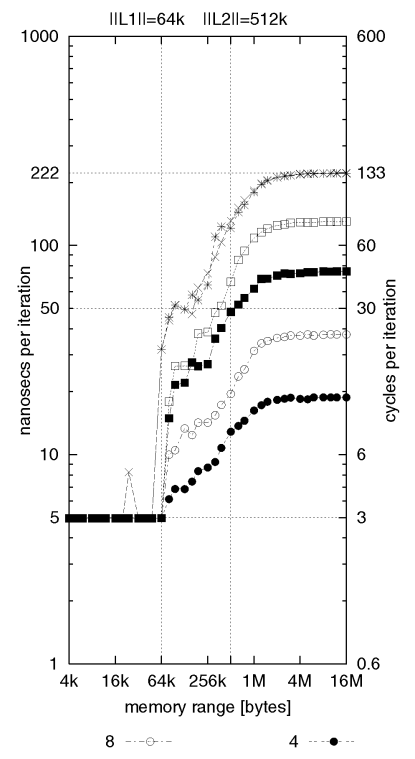

(d)

Fig. 5. Calibration tool: Cache sizes, line sizes, and latencies. (a) Origin2000, (b) Sun Ultra, (c) Intel PC, and (d) AMD PC.

\subsection{Calibration Tool}

In order to analyze the impact of memory access costs in detail, we need to know the characteristic parameters of the memory system, including memory sizes, cache sizes, cache line sizes, and access latencies. Often, not all of these parameters are (correctly) listed in the hardware manuals. In the following, we describe a simple but powerful calibration tool to measure the (cache) memory characteristics of an arbitrary machine.

\subsubsection{Calibrating the Memory System}

Our calibrator is a simple C program, mainly a small loop that executes a million memory reads. By changing the stride and the size of the memory area, we force varying cache miss rates. Thus, we can calculate the latency for a cache miss by comparing the execution time without misses to the execution time with exactly one miss per iteration. This approach only works if memory accesses are executed purely sequentially, i.e., we have to make sure that neither two or more load instructions nor memory access and pure CPU work overlap. We use a simple pointer chasing mechanism to achieve this: The memory area we access is initialized such that each load returns the address for the subsequent load in the next iteration. Thus, superscalar CPUs cannot benefit from their ability to hide memory access latency by speculative execution.

To measure the cache characteristics, we ran our experiment several times, varying the stride and the array size. We made sure that the stride varied at least between four bytes and twice the maximal expected cache line size and that the array size varied from half the minimal expected cache size to at least 10 times the maximal expected cache size.

Fig. 5a depicts the resulting execution time (in nanoseconds) per iteration for different array sizes on an Origin2000 (MIPS R10000, $250 \mathrm{MHz}=4$ ns per cycle). Each curve represents a different stride. From this figure, we can derive the desired parameters as follows: Up to an array size of 32 Kbytes, one iteration takes 8 nanoseconds (i.e., two cycles), independent of the stride. Here, no cache misses occur once the data is loaded as the array completely fits in L1 cache. One of the two cycles accounts for executing the load instruction and the other one accounts for the latency to access data in L1. With array sizes between 32 Kbytes and 4 Mbytes, the array exceeds L1, but still fits in L2. Thus, L1 misses occur. The miss rate (i.e., the number of misses per iteration) depends on the stride $(s)$ and the L1 cache line size $\left(L S_{L 1}\right)$. With $s<L S_{L 1}, \frac{s}{L S_{L 1}}$ L1 misses occur per iteration (or one L1 miss occurs every $\frac{L S_{L 1}}{s}$ iterations). With $s \geq L S_{L 1}$, each load causes an L1 miss. Fig. 5 shows that the execution time increases with the stride, up to a stride of 32. Then, it stays constant. Hence, L1 line size is 32 bytes. Further, L1 miss latency (i.e., L2 access latency) is $32 \mathrm{~ns}-8 \mathrm{~ns}=24 \mathrm{~ns}$, or six cycles. Similarly, when the array size exceeds L2 size (4 Mbytes), L2 misses occur. Here, the L2 line size is 128 bytes and the L2 miss latency (memory access latency) is $432 \mathrm{~ns}-32 \mathrm{~ns}=400 \mathrm{~ns}$, or 100 cycles. Analogously, Figs. 5b, 5c, and $5 d$ show the results for a Sun Ultra (Sun UltraSPARC, $200 \mathrm{MHz}=5 \mathrm{~ns}$ per cycle), an Intel PC (Intel Pentium III, $450 \mathrm{MHz}=2.22$ ns per cycle), and an AMD PC (AMD Athlon, $600 \mathrm{MHz}=1.67 \mathrm{~ns}$ per cycle).

The sequential memory bandwidth for our systems, listed in Table 1 , is computed from the cache line sizes and the latencies as follows:

$$
b w_{s e q}=\frac{L S_{L 2}}{l_{M e m}+\frac{L S_{L 2}}{L S_{L 1}} * l_{L 2}} .
$$

We will discuss parallel memory bandwidth in the next section.

\subsubsection{Calibrating the TLB}

We use a similar approach as above to measure TLB miss costs. The idea here is to force one TLB miss per iteration, 
TABLE 1

Calibrated Performance Characteristics

\begin{tabular}{|c|c|c|c|c|c|}
\hline & & SGI Origin2000 & Sun Ultra & Intel PC & AMD PC \\
\hline $\begin{array}{l}\text { OS } \\
\text { CPU } \\
\text { CPU speed } \\
\text { main-memory size }\end{array}$ & & $\begin{array}{c}\text { IRIX64 } 6.5 \\
\text { MIPS R10000 } \\
250 \mathrm{MHz} \\
48 \mathrm{~GB}(4 \mathrm{~GB} \text { local) }\end{array}$ & $\begin{array}{c}\text { Solaris } 2.5 .1 \\
\text { Sun UltraSparc } \\
200 \mathrm{MHz} \\
512 \mathrm{MB}\end{array}$ & $\begin{array}{c}\text { Linux } 2.2 .14 \\
\text { Intel PentiumlII } \\
450 \mathrm{MHz} \\
512 \mathrm{MB}\end{array}$ & $\begin{array}{c}\text { Linux } 2.2 .14 \\
\text { AMD Athlon } \\
600 \mathrm{MHz} \\
384 \mathrm{MB}\end{array}$ \\
\hline L1 cache size & $\|L 1\|$ & $32 \mathrm{~KB}$ & $16 \mathrm{~KB}$ & $16 \mathrm{~KB}$ & $64 \mathrm{~KB}$ \\
\hline L1 cache line size & $L S_{L 1}$ & 32 bytes & 16 bytes & 32 bytes & 64 bytes \\
\hline L1 cache lines & $|L 1|_{L 1}$ & 1024 & 1024 & 512 & 1024 \\
\hline L2 cache size & $\|L 2\|$ & $4 \mathrm{MB}$ & $1 \mathrm{MB}$ & $512 \mathrm{~KB}$ & $512 \mathrm{~KB}$ \\
\hline L2 cache line size & $L S_{L 2}$ & 128 bytes & 64 bytes & 32 bytes & 64 bytes \\
\hline L2 lines & $|L 2|_{L 2}$ & 32,768 & 16,384 & 16,384 & 8192 \\
\hline TLB entries & $|T L B|$ & 64 & 64 & 64 & 32 \\
\hline TLB $_{2}$ entries & $\left|T L B_{2}\right|$ & - & - & - & 256 \\
\hline page size & $\|P g\|$ & $32 \mathrm{~KB}$ & $8 \mathrm{~KB}$ & $4 \mathrm{~KB}$ & $4 \mathrm{~KB}$ \\
\hline TLB size $\quad(|T L B| *\|P g \mid\|)$ & $\|T L B\|$ & $2 \mathrm{MB}$ & $512 \mathrm{~KB}$ & $256 \mathrm{~KB}$ & $128 \mathrm{~KB}$ \\
\hline TLB $_{2}$ size $\left(\left|T L B_{2}\right| *\|P g\|\right)$ & $\left\|T L B_{2}\right\|$ & - & - & - & $1 \mathrm{MB}$ \\
\hline L1 miss latency & $l_{L 2}$ & $24 \mathrm{~ns}=6$ cycles & $30 \mathrm{~ns}=6$ cycles & $42.2 \mathrm{~ns}=19$ cycles & $45 \mathrm{~ns}=27$ cycles \\
\hline L2 miss latency & $l_{M e m}$ & $400 \mathrm{~ns}=100$ cycles & 195 ns $=39$ cycles & $93.3 \mathrm{~ns}=42$ cycles & $172 \mathrm{~ns}=103$ cycles \\
\hline TLB miss latency & $l_{T L B}$ & 228 ns $=57$ cycles & $270 \mathrm{~ns}=54$ cycles & $11.1 \mathrm{~ns}=5$ cycles & $8 \mathrm{~ns}=5$ cycles \\
\hline $\mathrm{TLB}_{2}$ miss latency & $l_{T L B_{2}}$ & - & - & - & 87 ns $=52$ cycles \\
\hline seq. memory bandwidth & $b w_{s e q}$ & $246 \mathrm{MB} / \mathrm{s}$ & $193 \mathrm{MB} / \mathrm{s}$ & $225 \mathrm{MB} / \mathrm{s}$ & $281 \mathrm{MB} / \mathrm{s}$ \\
\hline par. memory bandwidth & $b w_{p a r}$ & $555 \mathrm{MB} / \mathrm{s}$ & $244 \mathrm{MB} / \mathrm{s}$ & $484 \mathrm{MB} / \mathrm{s}$ & $670 \mathrm{MB} / \mathrm{s}$ \\
\hline
\end{tabular}

but to avoid any cache misses. We force TLB misses by using a stride that is larger than the system's page size and by choosing the array size such that we access more distinct spots than there are TLB entries. Cache misses will occur at least as soon as the number of spots accessed exceeds the number of cache lines. We cannot avoid that. But, even with fewer spots accessed, two or more spots might be mapped to the same cache line, causing conflict misses. To avoid this, we use strides that are not exactly powers of two, but slightly bigger, shifted by $\mathrm{L} 2$ cache line size, i.e., $s=2^{x}+L S_{L 2}$.

Fig. 6 shows the results for four machines. The X-axis now gives the number of spots accessed, i.e., array size divided by stride. Again, each curve represents a different stride. From Fig. 6a (Origin2000), for example, we derive the following: As above, we observe the base line of 8 nanoseconds (i.e., two cycles) per iteration. The smallest number of spots where the performance decreases due to TLB misses is 64, hence, there must be 64 TLB entries. The decrease at 64 spots occurs with strides of 32 Kbytes or more, thus, the page size is 32 Kbytes. Further, TLB miss latency is 236 ns -8 ns $=228$ ns, or 57 cycles. Fig. 6 d correctly reflects the Athlon's two TLB levels with 32 and 256 entries, respectively. The third step in the curves at 1,024 spots is caused by L1 misses as L1 latency is five times higher than TLB latency on the Athlon. The same holds for the second

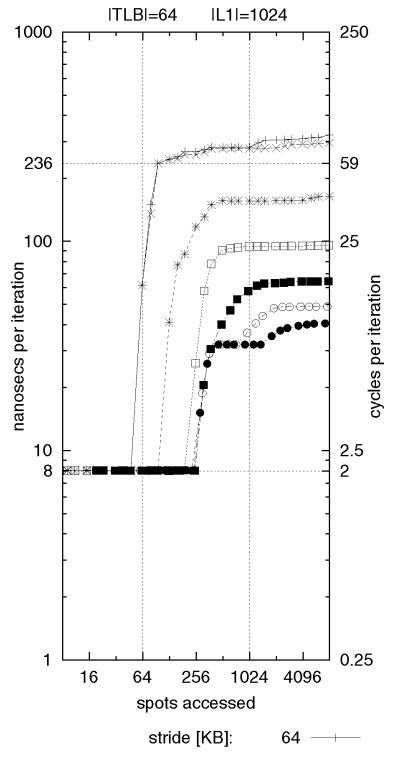

(a)

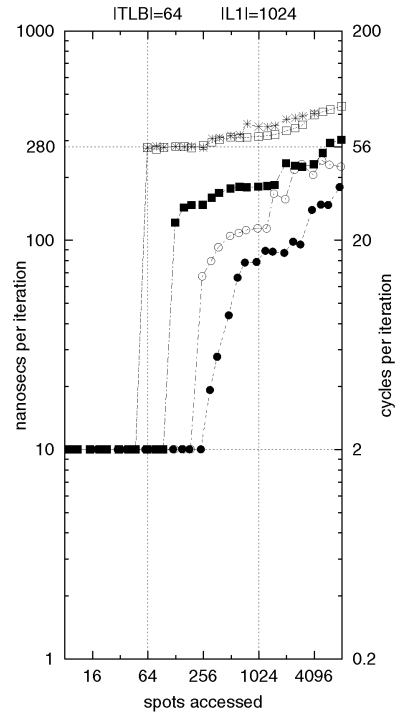

32

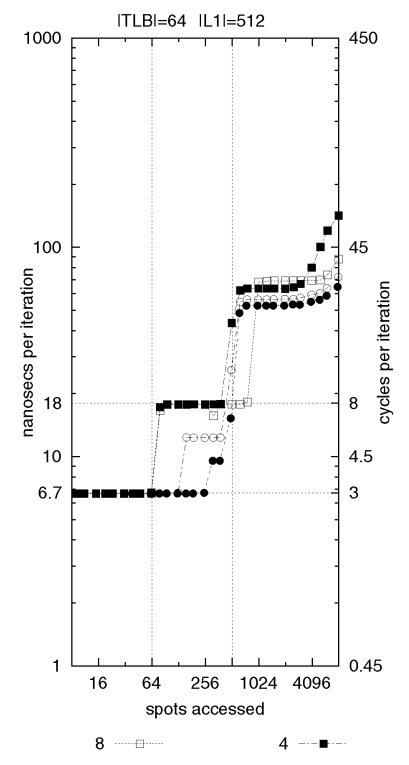

(c)

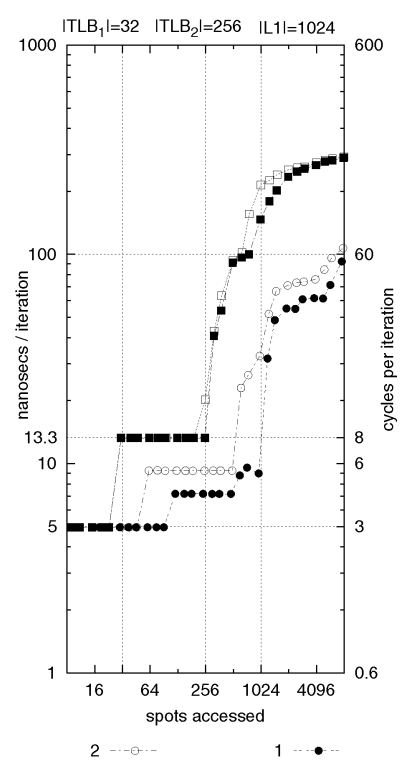

(d)

Fig. 6. Calibration tool: TLB entries and TLB miss costs. (a) Origin2000, (b) Sun Ultra, (c) Intel PC, and (d) AMD PC. 


\begin{tabular}{|c|c|c|}
\hline normal loop & multi-cursor & prefetch \\
\hline $\begin{array}{l}\text { for }(\text { int } \text { tot }=\mathrm{i}=0 ; \mathrm{i}<\mathrm{N} ; \mathrm{i}++)\{ \\
\quad \text { tot }+=\text { buf }[\mathrm{i}] \\
\}\end{array}$ & $\begin{array}{l}\text { for }\left(\text { int } \text { tot }_{0}=\operatorname{tot}_{1}=\mathrm{i}=0, \mathrm{C}=\mathrm{N} / 2 ; \mathrm{i}<\mathrm{C} ; \mathrm{i}+\mathrm{H}\right)\{ \\
\quad \operatorname{tot}_{0}+=\text { buf }[\mathrm{i}] ; \\
\text { tot }_{1}+=\text { buf }[\mathrm{i}+\mathrm{C}] ; \\
\} \text { int tot }=\text { tot }_{0}+\text { tot }_{1} ;\end{array}$ & $\begin{array}{l}\text { for }(\text { int tot }=\mathrm{i}=0 ; \mathrm{i}<\mathrm{N} ; \mathrm{i}+\mathrm{H})\{ \\
\# \text { prefetch buf }[\mathrm{i}+32] \text { freq }=32 \\
\quad \text { tot }+=\text { buf }[\mathrm{i}]\end{array}$ \\
\hline 5.88 cycles/addition & 3.75 cycles/addition & $3.88 \rightarrow 2$ cycles/addition \\
\hline
\end{tabular}

Fig. 7. Three ways to add a buffer of intergers and costs per addition on the Origin2000.

step in the Pentium III curves (Fig. 6c) at 512 spots. On the Origin2000 and on the Sun, L1 misses also occur with more than 1,024 spots accessed, but their impact is negligible as TLB latency is almost 10 times higher than L1 latency on these machines.

Table 1 gathers the results for all four machines. The PCs have the highest L2 latency, probably as their L2 caches are running at only half the CPUs' clock speed, but they have the lowest memory latency. Their very low TLB latency is due to the hardware implementation of TLB management on the PCs, which avoids the costs of trapping to the operating system on a TLB miss, which is necessary in the software-controlled TLBs of the other systems. The Origin2000 has the highest memory latency, but, due to its large cache lines, it achieves better sequential memory bandwidth than the Sun and the Intel PC.

\subsection{Parallel Memory Access}

It is interesting to note that the calibrated latencies in Table 1 do not always confirm the suggested latencies in the sequential scan experiment from Fig. 4. For the Pentium III, the access costs per memory read of $52 \mathrm{~ns}$ at a stride of 32 bytes and 204 ns at a stride of 128 bytes for the Origin2000, are considerably lower than their memory latencies (135 ns, respectively, $424 \mathrm{~ns}$ ), whereas, in the case of the Sun Ultra, the scan measurement at L2 line size almost coincides with the calibrated memory latency. The discrepancies are caused by parallel memory access which can occur on CPUs that feature both speculative execution and a nonblocking memory system. This allows a CPU to execute multiple memory load instructions in parallel, potentially enhancing memory bandwidth above the level of cache-line size divided by latency. Prerequisites for this technique are a bus system with excess transport capacity and a nonblocking cache system that allows multiple outstanding cache misses.

To answer the question about what needs to be done by an application programmer to achieve these parallel memory loads, let us consider a simple programming loop that sums an array of integers. Fig. 7 shows three implementations, where the leftmost column contains the standard approach that results in sequential memory loads into the buf[size] array. An R10000 processor can continue executing memory load instructions speculatively until four of them are stalled. In this loop, that will indeed happen if buf[i], buf[i+1], buf[i+2], and buf[i+3] are not in the (L2) cache. However, due to the fact that our loop accesses consecutive locations in the buf array, these four memory references request the same 128-byte L2 cache line. Consequently, no parallel memory access takes place. If we assume that this loop takes two cycles per iteration, ${ }^{3}$ we can calculate that 32 iterations cost $32^{*} 2$ $+124=188$ cycles (where 124 is the memory latency on our Origin2000); a total mean cost of 5.88 cycles per addition.

Parallel memory access can be enforced by having one loop that iterates two cursors through the buf[size] array (see the middle column of Fig. 7). This causes two parallel 128 byte (=32 integer) L2 cache line fetches from memory per 32 iterations, for a total of 64 additions. On the R10000, the measured maximum memory bandwidth of the bus is 555 Mbytes, so fetching two 128-byte cache lines in parallel costs only 112 cycles (instead of $124+124$ ). The mean cost per addition is, hence, $2+112 / 64=3.75$ cycles.

It is important to note that parallel memory access is achieved only if the ability of the CPU to execute multiple instructions speculatively spans multiple memory references in the application code. In other words, the parallel effect disappears if there is too much CPU work between two memory fetches (more than 124 cycles on the R10000) or if the instructions are interdependent, causing a CPU stall before reaching the next memory reference. For database algorithms, this means that random access operations like hashing will not profit from parallel memory access as following a linked list (hash bucket chain) causes one iteration to depend on the previous, hence, a memory miss will block execution. Only sequential algorithms with CPU processing costs less than the memory latency will profit as in the simple scan experiment from Fig. 4. This experiment reaches optimal parallel bandwidth when the stride is equal to this L2 cache line size. As each loop iteration then requests one subsequent cache line, modern CPUs will have multiple memory loads outstanding, executing them in parallel. Results are summarized at the bottom of Table 1, showing the parallel effect to be especially strong on the Origin2000, the Pentium III, and the Athlon. In other words, if the memory access pattern is not sequential (as in equijoin), the memory access penalty paid on these systems is actually much higher than suggested by Fig. 4, but determined by the latencies from Table 1.

\subsection{Prefetched Memory Access}

Computer systems with a nonblocking cache can shadow memory latency by performing a memory fetch well before it is actually needed. CPUs like the R10000, the Pentium III, the Athlon, and the newer SPARC Ultra2 models have special prefetching instructions for this purpose. These instructions can be thought of as memory

3. As each iteration of our loop consists of a memory load (buf[i]), an integer addition (of "total" with this value), an integer increment (of i), a comparison, and a branch, the R10000 manual suggests a total cost of, minimally, six cycles. However, due to the speculative execution in the $\mathrm{R} 10000$ processor, this is reduced to two cycles on average. 
load instructions that do not deliver a result. Their only side effect is a modification of the status of the caches. Mowry describes compiler techniques to generate these prefetching instructions automatically [1]. These techniques optimize array accesses from within loops when most loop information and dependencies are statically available and, as such, are very appropriate for scientific code written in Fortran. Database code written in $\mathrm{C} / \mathrm{C}++$, however, does not profit from these techniques as even the most simple table scan implementation will typically result in a loop with both a dynamic stride and length as these are (dynamically) determined by the width and length of the table that is being scanned. Also, if table values are compared or manipulated within the loop using a function call (e.g., comparing two values for equality using a $C$ function looked up from some ADT table or a $\mathrm{C}++$ method with late binding), the unprotected pointer model of the $\mathrm{C} / \mathrm{C}++$ languages forces the compiler to consider the possibility of side effects from within that function, eliminating the possibility of optimization.

In order to provide the opportunity to still enforce memory prefetching in such situations, the MipsPRO compiler for the R10000 systems of Silicon Graphics allows passing of explicit prefetching hints by use of pragmas, as depicted in the rightmost column of Fig. 7. This pragma tells the compiler to request the next cache line once in every 32 iterations. Such a prefetch-frequency is generated by the compiler by applying loop unrolling (it unrolls the loop 32 times and inserts one prefetch instruction). By hiding the memory prefetch behind 64 cycles of work, the mean cost per addition in this routine is reduced to $2+$ $((124-64) / 32)=3.88$ cycles. Optimal performance is achieved in this case when prefetching two cache lines ahead every 32 iterations (\#prefetch buf[i+64] freq = 32). The 124 cycles of latency are then totally hidden behind 128 cycles of CPU work and a new cache line is requested every 64 cycles. This setting effectively combines prefetching with parallel memory access (two cache lines in 128 cycles instead of 248) and reduces the mean cost per addition to the minimum two cycles, three times faster than the simple approach.

\subsection{Future Hardware Features}

In spite of memory latency staying constant, hardware manufacturers have been able to increase memory bandwidth in line with the performance improvements of CPUs by working with ever wider lines in the L1 and L2 caches. As cache lines grew wider, buses also did. The latest Sun Ultra II workstations, for instance, have a 64-byte L2 cache line which is filled in parallel using a 576-bit wide PCI bus ( $576=64^{*} 8$ plus 64 bits overhead). The strategy of doubling memory bandwidth by doubling the number of DRAM chips and bus lines is now seriously complicating system board design. The future Rambus [30] memory standard eliminates this problem by providing a "protocol-driven memory bus." Instead of designating one bit in the bus for one bit of data transported to the cache line, this new technology serializes the DRAM data into packets using a protocol and sends these packets over a thin (16-bit) bus that runs at very high speeds (up to $800 \mathrm{MHz}$ ). While this allows for continued growth in memory bandwidth, it does not provide the same perspective for memory latency as Rambus still needs to access DRAM chips and there will still be the relatively long distance for the signals to travel between the CPU and these memory chips on the system board, both factors ensuring a fixed startup cost (latency) for any memory traffic.

A radical way around the high latencies mandated by off-CPU DRAM systems is presented in the proposal to integrate DRAM and CPU in a single chip called IRAM (Intelligent RAM) [31]. Powerful computer systems could then be built using many such chips. Finding a good model for programming such a highly parallel system seems one of the biggest challenges of this approach. Another interesting proposal worth mentioning here is "smarter memory" [32], which would allow the programmer to give a "cache-hint" by specifying the access pattern that is going to be used on a memory region in advance. This way, the programmer is no longer obliged to organize his data structures around the size of a cache line. Instead, the cache adapts its behavior to the needs of the application. Such a configurable system is, in some sense, a protocol-driven bus system, so Rambus is a step in this direction. However, both configurable memory access and IRAM have not yet been implemented in custom hardware, let alone in OS and compiler tools that would be needed to program them usefully.

Recent developments concerning memory caches are to move the L2 cache closer to the CPU, either locating it on the same multichip module (e.g., Intel's first Pentium III, "Katmai," or AMD's first Athlon generation) or even including it on the CPU's die (e.g., Intel's latest Pentium III, "Coppermine," or AMD's latest Athlon "Thunderbird"). While reducing L2 latency-the L2 caches now operate at half or even full CPU speed-these trends do not reduce the memory latency. Further, on-chip caches are usually smaller than off-chip caches and, hence, provide even less potential to avoid memory accesses. Similarly, additional L3 caches-although increasing the total cache capacity-cannot reduce memory latency, but rather might even increase it due to an increased management overhead.

Concerning CPU technology, it is anticipated [33] that the performance advances dictated by Moore's law will continue well into the millennium. However, performance increase will also be brought by more parallelism within the CPU. The upcoming IA-64 architecture has a design called Explicitly Parallel Instruction Computing (EPIC) [34] which allows instructions to be combined in bundles, explicitly telling the CPU that they are independent. The IA-64 is specifically designed to be scalable in the number of functional units, so, while newer versions are released, more and more parallel units will be added. This means that, while current PC hardware uses less parallel CPU execution than the RISC systems, this will most probably change in the new 64-bit PC generation.

Summarizing, we have identified the following ongoing trends in modern hardware:

- CPU performance will keep growing with Moore's law for years to come. 


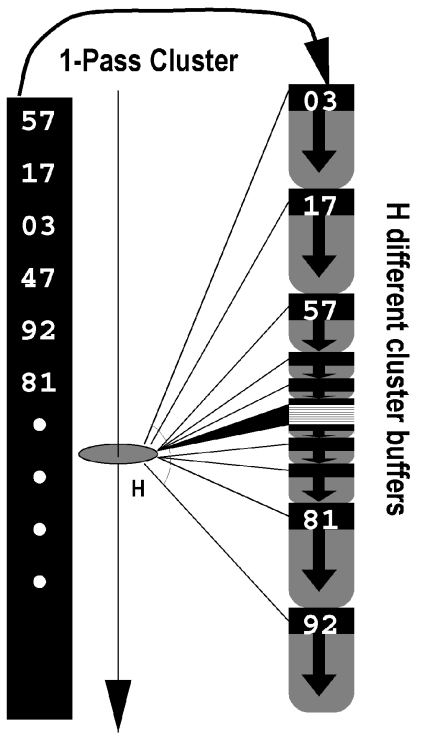

Fig. 8. Straightforward clustering algorithm.

- A growing part of this performance increase will come from parallelism within the CPU.

- New bus technology will provide sufficient growth in memory bandwidth.

- Memory latency will not improve significantly.

This means that the failure of current DBMS technology to properly exploit memory and CPU resources of modern hardware [2], [4], [3], [5] will grow worse. Modern database architecture should therefore take these new hardware issues into account. With this motivation, we investigate the following new approaches to large main-memory equi-joins that are specifically aimed at optimizing resource utilization of modern hardware.

\section{Partitioned Hash-Join}

Shatdal et al. [12] showed that a main-memory variant of Grace Join in which both relations are first partitioned on hash-number into $H$ separate clusters fit the memory cache and performs better than normal bucket-chained hash join. This work employs a straightforward clustering-algorithm that simply scans the relation to be clustered once, inserting each scanned tuple in one of the clusters, as depicted in Fig. 8. This constitutes a random access pattern that writes into $H$ separate locations. If $H$ is too large, there are two factors that degrade performance. First, if $H$ exceeds the number of TLB entries, ${ }^{4}$ each memory reference will become a TLB miss. Second, if $H$ exceeds the number of available cache lines (L1 or L2), cache thrashing occurs, causing the number of cache misses to explode.

As an improvement over this straightforward algorithm, we propose a clustering algorithm that has a memory access pattern that requires less random-access, even for high values of $H$.

4. If the relation is very small and fits the total number of TLB entries times the page size, multiple clusters will fit into the same page and this effect will not occur.

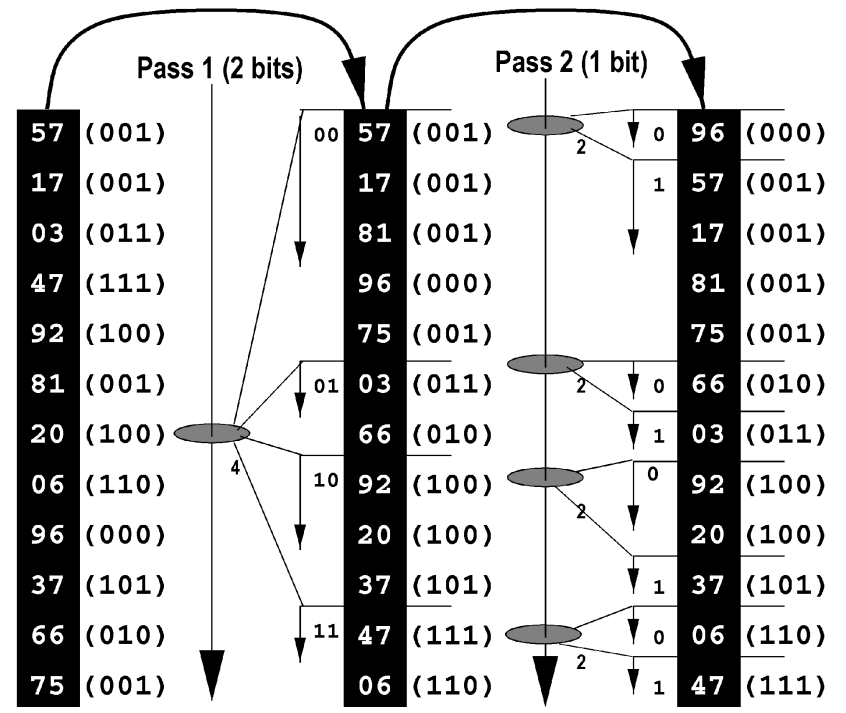

Fig. 9. Two-pass/3-bit radix cluster (lower bits indicated between parentheses).

\subsection{Radix-Cluster Algorithm}

The radix-cluster algorithm divides a relation into $H$ clusters using multiple passes (see Fig. 9). Radix-clustering on the lower $B$ bits of the integer hash-value of a column is achieved in $P$ sequential passes in which each pass clusters tuples on $B_{p}$ bits, starting with the leftmost bits $\left(\sum_{1}^{P} B_{p}=B\right)$. The number of clusters created by the radix-cluster is $H=\prod_{1}^{P} H_{p}$ where each pass subdivides each cluster into $H_{p}=2^{B_{p}}$ new ones. When the algorithm starts, the entire relation is considered one single cluster and is subdivided into $H_{1}=$ $2^{B_{1}}$ clusters. The next pass takes these clusters and subdivides each into $H_{2}=2^{B_{2}}$ new ones, yielding $H_{1} * H_{2}$ clusters in total, etc. Note that, with $P=1$, radix-cluster behaves like the straightforward algorithm.

For ease of presentation, we did not use a hash function in the table of integer values displayed in Fig. 9. In practice, though, it is better to use such a function, even on integers, in order to ensure that all bits of the table values play a role in the lower bits of the radix number.

The interesting property of the radix-cluster is that the number of randomly accessed regions $H_{x}$ can be kept low, while a high overall number of $H$ clusters can still be achieved using multiple passes. More specifically, if we keep $H_{x}=2^{B_{x}}$ smaller than the number of cache lines and the number of TLB entries, we totally avoid both TLB and cache thrashing.

After radix-clustering a column on $B$ bits, all tuples that have the same $B$ lowest bits in its column hash-value appear consecutively in the relation, typically forming chunks of $C / 2^{B}$ tuples (with $C$ denoting the cardinality of the entire relation). Therefore, it is not strictly necessary to store the cluster boundaries in some additional data structure; an algorithm scanning a radix-clustered relation can determine the cluster boundaries by looking at these lower $B$ "radix-bits." This allows very fine clusterings without introducing overhead by large boundary structures. It is interesting to note that a radix-clustered relation is in fact ordered on radix-bits. When using this algorithm in the partitioned hash-join, we exploit this property by 
TABLE 2

Hardware Counters Used for Execution Time Breakdown

\begin{tabular}{|c|c|c|c|c|}
\hline category & MIPS R10k & Sun UltraSPARC & Intel PentiumIII & AMD Athlon \\
\hline $\begin{array}{l}\text { memory } \\
\text { access }\end{array}$ & $\begin{array}{l}\text { L1_data_misses * 6cy } \\
\text { L2_data_misses * 100cy } \\
\text { TLB_misses * 57cy } \\
\text { L1_inst_misses * 6cy } \\
\text { L2_inst_misses * 100cy }\end{array}$ & $\begin{array}{l}\text { DC_misses }{ }^{1 * 6 c y} \\
\text { EC_misses }^{2} * 39 c y \\
M_{T L B} * 54 c y \\
\text { stall_IC_miss }\end{array}$ & $\begin{array}{l}\text { DCU_miss_outstanding } \\
M_{T L B} * 5 c y \\
\text { IFU_mem_stall } \\
\text { ITLB_miss } * 32 \mathrm{cy}^{3}\end{array}$ & $\begin{array}{l}\text { DC_refills_from_L2 } * 27 c y \\
\text { DC_refills_from_system * } 103 c y \\
\text { L1_DTLB_misses } * 5 c y \\
\text { L2_DTLB_misses * 52cy } \\
\text { IC_misses } * 27 c y \\
\text { L1_ITLB_misses } * 5 c y \\
\text { L2_ITLB_misses } * 52 c y\end{array}$ \\
\hline $\begin{array}{l}\text { CPU } \\
\text { stalls }\end{array}$ & branches_mispredicted $* 4 c y$ & $\begin{array}{l}\text { stall_mispred } \\
\text { stall_fpdep }\end{array}$ & $\begin{array}{l}\text { br_miss_pred }{ }^{*} 17 \mathrm{cy}^{3} \\
\text { ILD_stalled } \\
\text { resource_stalls } \\
\text { partial_rat_stalls }\end{array}$ & branches_mispredicted \\
\hline divisions & $C * 2 * 35$ cy & $C * 2 * 60$ су & cycles_div_busy & $C * 2 * 40$ cy \\
\hline
\end{tabular}

performing a merge step on the radix-bits of both radixclustered relations to get the pairs of clusters that should be hash-joined with each other.

\subsection{Quantitative Assessment}

The radix-cluster algorithm presented in the previous section provides three tuning parameters:

1. the number of radix-bits used for clustering $(B)$, implying the number of clusters $H=2^{B}$,

2. the number of passes used during clustering $(P)$, and

3. the number of radix-bits used per clustering pass $\left(B_{p}\right)$.

In the following, we present an exhaustive series of experiments to analyze the performance impact of the different settings of these parameters. After establishing which parameter settings are optimal for radix-clustering a relation on $B$ radix-bits, we turn our attention to the performance of the join algorithm with varying values of $B$. For both phases, clustering and joining, we investigate how appropriate implementations techniques can improve the performance even further. Finally, these two experiments are combined to gain insight in the overall join performance.

\subsubsection{Experimental Setup}

In our experiments, we use binary relations (BATs) of 8 bytes wide tuples and varying cardinalities $(C)$, consisting of uniformly distributed random numbers. Each value occurs three times. Hence, in the joinexperiments, the join hit-rate is three. The result of a join is a BAT that contains the [OID, OID] combinations of matching tuples (i.e., a join-index [35]). Subsequent tuple reconstruction is cheap in Monet and equal for all algorithms, so, just as in [12], we do not include it in our comparison. The experiments were carried out on the machines presented in Section 2.3, an SGI Origin2000, a Sun Ultra, an Intel PC, and an AMD PC (cf. Table 1).
To analyze the performance behavior of our algorithms in detail, we break down the overall execution time into the following major categories of costs:

Memory access. In addition to memory access costs for data as analyzed above, this category also contains memory access costs caused by instruction cache misses.

CPU stalls. Beyond memory access, there are other events that make the CPU stall similar to branch mispredictions or other so-called resource related stalls.

Divisions. We treat integer divisions separately as they play a significant role in our hash-join (see below).

Real CPU. This is the remaining time, i.e., the time the CPU is indeed busy executing the algorithms.

The four architectures we investigate provide different hardware counters [26] that enable us to measure each of these cost factors accurately. Table 2 gives an overview of the counters used. Some counters yield the actual CPU cycles spent during a certain event, others just return the number of events that occurred. In the latter case, we multiply the counters by the penalties of the events (as calibrated in Section 2.3). None of the architectures provides a counter for the pure CPU activity. Hence, we subtract the cycles spent on memory access, CPU stalls, and integer division from the overall number of cycles and assume that the rest are pure CPU costs.

In our experiments, we found that, in our algorithms, branch mispredictions and instruction cache misses do not play a role on either architecture. The reason is that, in contrast to most commercial DBMSs, Monet's code base is designed for efficient main-memory processing. Monet uses a very large grain size for buffer management in its operators (an entire BAT), therefore processing exhibits much code locality during execution and, hence, avoids instruction cache misses and branch mispredictions. Thus, for simplicity of presentation, we omit these events in our evaluation. 


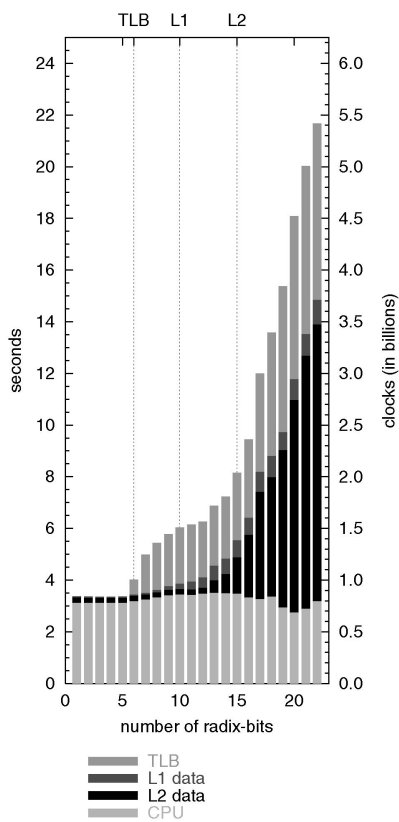

(a)

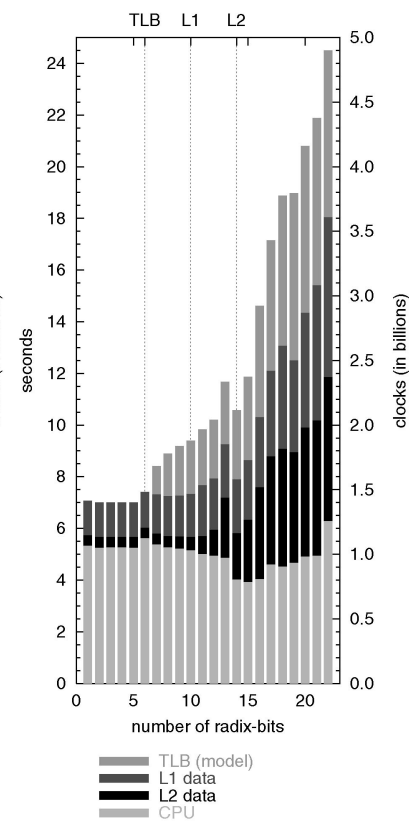

(b)

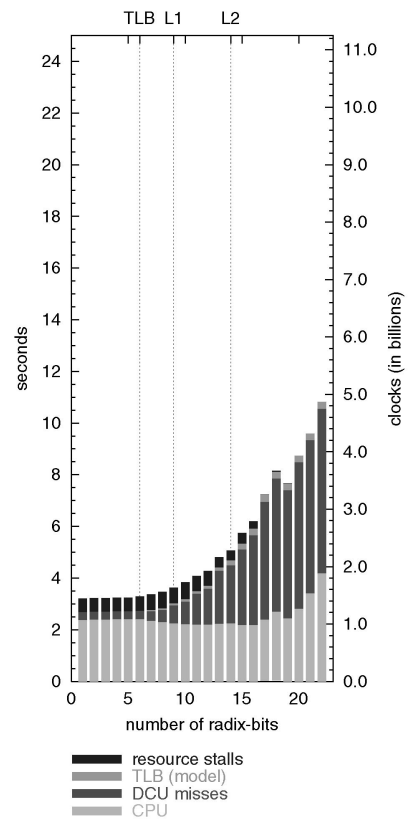

(c)

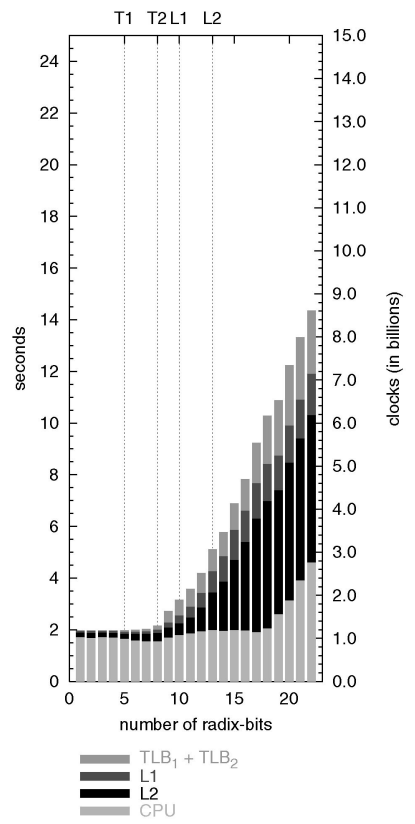

(d)

Fig. 10. Execution time breakdown of radix-cluster using one pass (Cardinality= 8 M). (a) Origin2000, (b) Sun Ultra, (c) Intel PC, and (d) AMD PC.

\subsubsection{Radix Cluster}

To analyze the impact of all three parameters $\left(B, P, B_{p}\right)$ on radix clustering, we conduct two series of experiments, keeping one parameter fixed and varying the remaining two.

First, we conduct experiments with various numbers of radix-bits and passes, distributing the radix-bits evenly across the passes. Fig. 10 shows an execution time breakdown for 1-pass radix-cluster $(C=8 \mathrm{M})$ on each architecture. The pure CPU costs are nearly constant across all numbers of radix-bits, taking about 3 seconds on the Origin, 5.5 seconds on the Sun, 2.5 seconds on the Pentium III, and about 1.7 seconds on the Athlon. Memory and TLB costs are low with small numbers of radix-bits, but grow significantly with the rising numbers of radix-bits. With more than 6 radix-bits, the number of clusters to be filled concurrently exceeds the number of TLB entries (64), causing the number of TLB misses to increase significantly. On the Origin and on the Sun, the execution time increases significantly due to their rather high TLB miss penalties. On the Pentium III, however, the impact of TLB misses is hardly visible due to its very low TLB miss penalty. The same holds for $\mathrm{TLB}_{1}$ misses on the Athlon, while the impact of the more expensive $\mathrm{TLB}_{2}$ misses is clearly visible. Analogously, the memory costs increase as soon as the number of clusters exceeds the number of L1 and L2 cache lines, respectively. Further, on the Pentium III, "resource related stalls" (i.e., stalls due to functional unit unavailability) play a significant role. They make up one-fourth of the execution time when the memory costs are low. When the memory costs rise, the resource related stalls decrease and finally vanish completely, reducing the impact of the memory penalty. In other words, minimizing the memory access costs does not fully pay back on the Pentium III as the resource related stalls partly take over their part. The Athlon, however, does not seem to suffer from such "resource related stalls."
Fig. 11 depicts the breakdown for radix-cluster using the optimal number of passes. The idea of multipass radixcluster is to keep the number of clusters generated per pass low-and, thus, the memory costs-at the expense of increased CPU costs. Obviously, the CPU costs are too high to avoid the TLB costs by using two passes with more than 6 radix-bits. Only with more than 15 radix-bits-i.e., when the memory costs exceed the CPU costs-will two passes win over one pass. The only exception is the Athlon, where multipass radix-cluster benefits from the high clock speed and, hence, two passes outperform one pass already from 11 radix-bits onward.

The only way to improve this situation is to reduce the CPU costs. Fig. 12 shows the source code of our radix-cluster routine. It performs a single-pass clustering on the $D$ bits that start $R$ bits from the right (multipass clustering in $P>1$ passes on $B=P * D$ bits is done by making subsequent calls to this function for pass $p=1$ through $p=P$ with parameters $D_{p}=D$ and $R_{p}=(p-1) * D$, starting with the input relation and using the output of the previous pass as input for the next). As the algorithm itself is already very simple, improvement can only be achieved by means of implementation techniques. We replaced the generic ADT-like implementation with a specialized one for each data type. Thus, we could inline the hash function and replace the memcpy by a simple assignment, saving two function calls per iteration.

Fig. 13 shows the execution time breakdown for the optimized multipass radix-cluster. The CPU costs have reduced significantly by almost a factor 4 . Replacing the two function calls has two effects. First, some CPU cycles are saved. Second, the CPUs can benefit more from the internal parallel capabilities using speculative execution as the code has become simpler and parallelization options more predictable. On the Pentium III, the resource stalls have doubled, neutralizing the CPU improvement partly. We think the simple loop does not consist of enough 


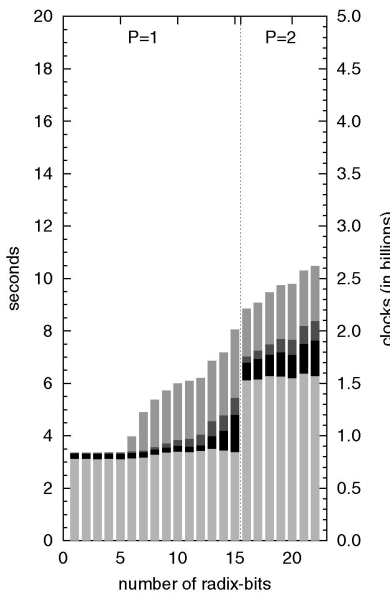

(a)

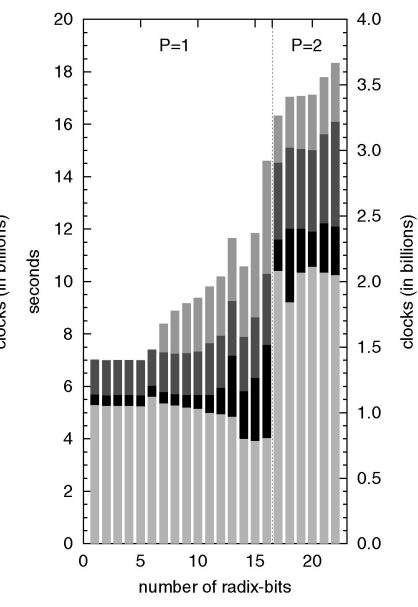

(b)

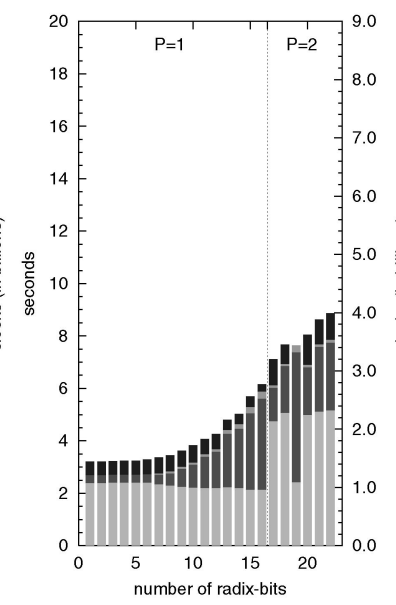

(c)

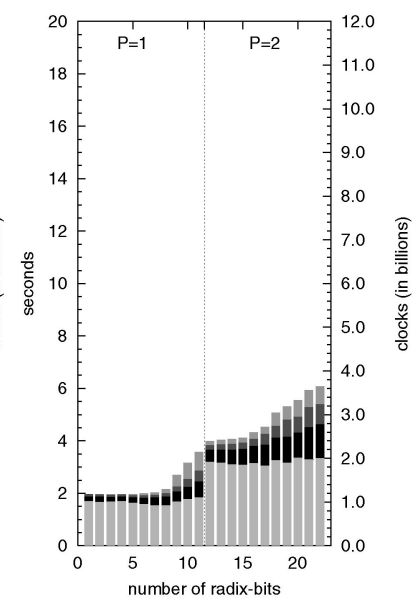

(d)

Fig. 11. Execution time breakdown of radix-cluster using an optimal number of passes $(C=8 \mathrm{M})$. (a) Origin2000, (b) Sun Ultra, (c) Intel PC, and (d) AMD PC.

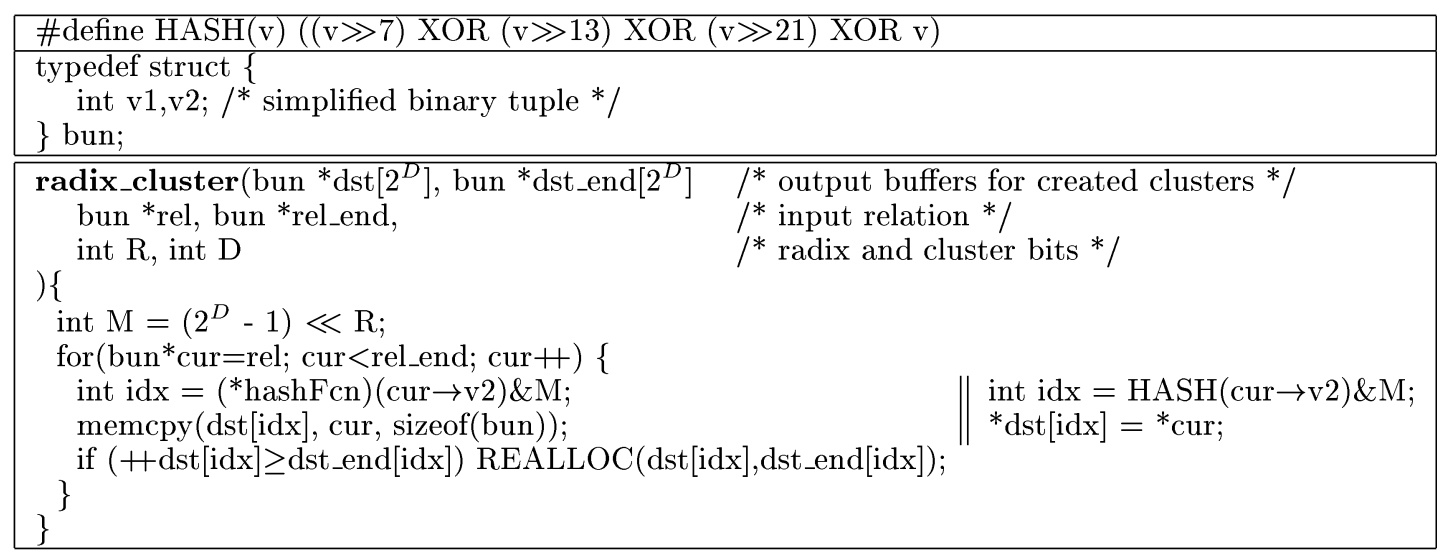

Fig. 12. C language radix-cluster with annotated CPU optimizations (right).

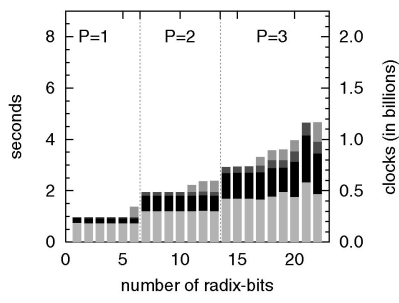

(a)

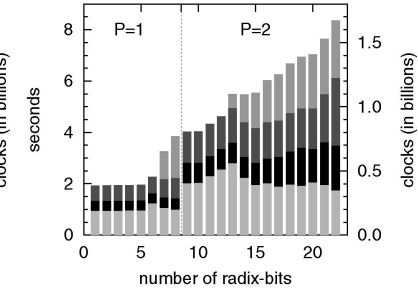

(b)

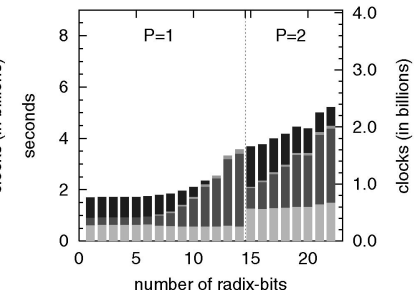

(c)

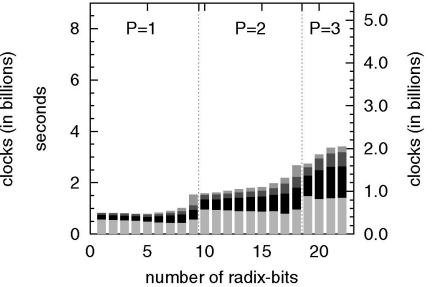

(d)

Fig. 13. Execution time breakdown of optimized radix-cluster using optimal number of passes $(C=8 \mathrm{M})$. (a) Origin2000, (b) Sun Ultra, (c) Intel PC, and (d) AMD PC.

instructions to fill the relatively long pipelines of the Pentium III efficiently.

With this optimization, multipass radix-cluster is already feasible with smaller numbers of radix-bits. On the Origin, two passes win with more than six radix-bits, and three passes win with more than 13 radix-bits, thus avoiding TLB thrashing nearly completely. Analogously, the algorithm creates at most 512 clusters per pass on the AMD PC, avoiding L1 thrashing, which is expensive due to the rather high L1 miss penalty on the Athlon. For the Pentium III, however, the improvement is marginal. The severe impact of resource stalls with low numbers of radix-bits makes the memory optimization of multipass radix-cluster almost ineffective.

In order to estimate the performance of radix-cluster and, especially, to predict the number of passes to be used for a certain number of radix-bits, we now provide an accurate cost model for radix-cluster. The cost model takes the number of passes, the number of radix-bits, and the cardinality as input and estimates the number of memory related events, i.e., L1 cache misses, L2 cache misses, and TLB misses. The overall execution time is calculated by scoring the events with their penalties and adding the pure CPU costs. 


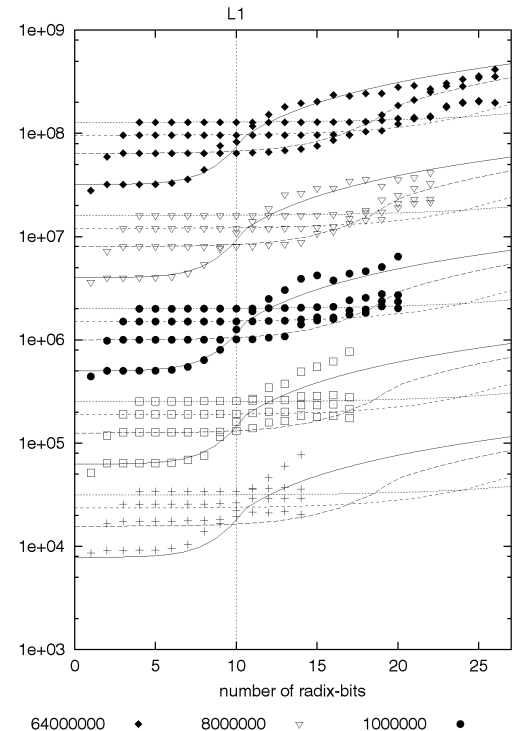

(a)

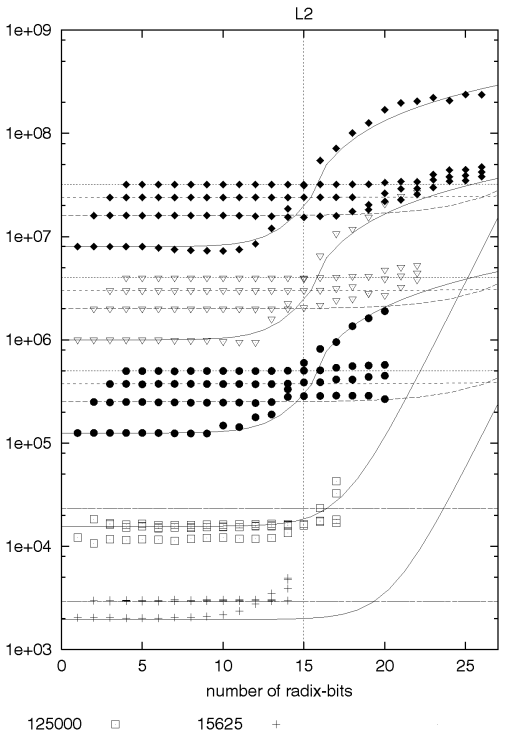

(b)

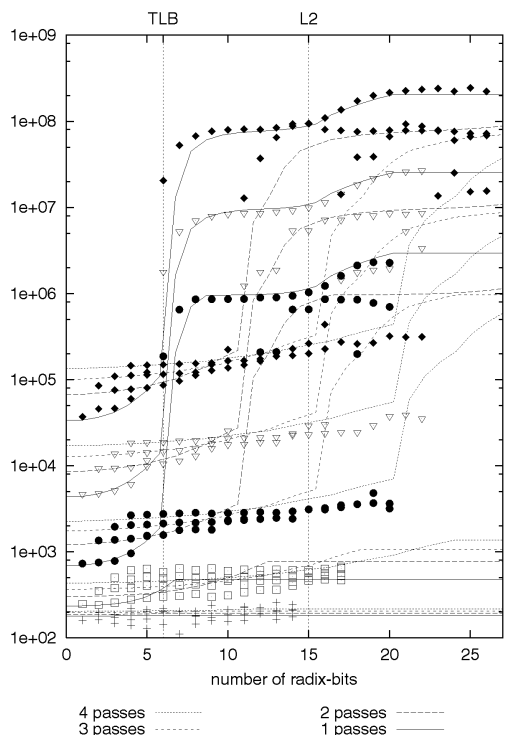

(c)

Fig. 14. Measured (points) and modeled (lines) events of radix-cluster (Origin2000). (a) L1 misses, (b) L2 misses, and (c) TLB misses.

$$
\begin{aligned}
T_{c}(P, B, C)=P *\left(C * w_{c}\right. & +M_{L 1, c}\left(\frac{B}{P}, C\right) * l_{L 2} \\
& +M_{L 2, c}\left(\frac{B}{P}, C\right) * l_{M e m} \\
& \left.+M_{T L B, c}\left(\frac{B}{P}, C\right) * l_{T L B}\right)
\end{aligned}
$$

with

$$
\begin{aligned}
& M_{L i, c}\left(B_{p}, C\right)= \\
& 2 *|R e|_{L i}+\left\{\begin{array}{l}
C * \frac{H_{p}}{|L i|_{L i}} * \min \left\{1, \frac{|R e|_{L i}}{|L i|_{L i}}\right\} \\
\text { if } \min \left\{H_{p},|R e|_{L i}\right\} \leq|L i|_{L i} \\
C * \min \left\{3,1+\log \left(\frac{H_{p}}{|L i|_{L i}}\right)\right\}, \\
\text { else }
\end{array}\right.
\end{aligned}
$$

and

$$
\begin{aligned}
& M_{T L B, c}\left(B_{p}, C\right)= \\
& 2 *|R e|_{P g}+\left\{\begin{array}{l}
|R e|_{P g} *\left(\frac{\min \left\{H_{p},|R e|_{P g}\right\}}{|T L B|}\right), \\
\text { if } \min \left\{H_{p},|R e|_{P g}\right\} \leq|T L B| \\
C *\left(1-\frac{|T L B|}{\min \left\{H_{p},|R e|_{P g}\right\}}\right), \\
\text { else }
\end{array}\right. \\
& \text { (if }|R e|_{P g}>|T L B| \\
& +\left\{\begin{array}{l}
C *\left(\frac{H_{p}}{|L 2|_{L 2}}\right) \\
\text { if } H_{p} \leq|L 2|_{L 2} \\
C * \min \left\{2,1+\log \left(\frac{H_{p}}{|L 2|_{L 2}}\right)\right\}, \\
\text { else. }
\end{array}\right.
\end{aligned}
$$

$|R e|_{L i}$ and $|C l|_{L i}$ denote the number of cache lines per relation and cluster, respectively, $|R e|_{P g}$ the number of pages per relation, $|L i|_{L i}$ the total number of cache lines, both for the L1 $(i=1)$ and L2 $(i=2)$ caches, and $|T L B|$ the number of TLB entries. $w_{c}$ denotes the pure CPU costs per tuple. To calibrate $w_{c}$, we reduced the cardinality so that all data fits in L1 and preloaded the input relation. Thus, we avoided memory access completely. We measured $w_{c}=100 \mathrm{~ns}$ on the Origin2000, $w_{c}=200 \mathrm{~ns}$ on the Sun, $w_{c}=180 \mathrm{~ns}$ on the Intel PC (including resource stalls), and $w_{c}=75 \mathrm{~ns}$ on the AMD PC.

The first term of $M_{L i, c}$ equals the minimal number of $\mathrm{L} i$ misses per pass for fetching the input and storing the output. The second term counts the number of additional $\mathrm{L} i$ misses when the number of distinct $\mathrm{L} i$ lines accessed concurrently (i.e., $\left.x=\min \left\{H_{p},|R e|_{L i}\right\}\right)^{5}$ either approaches the number of available $L i$ lines $\left(x \leq|L i|_{L i}\right)$ or even exceeds this. First, the probability that the requested cluster is not in the cache-due to address conflicts-increases until $H_{p}=|L i|_{L i}$. Then, the cache capacity is exhausted and a cache miss for each tuple to be assigned to a cluster is certain. But, with further increasing $H_{p}$, the number of cache misses also increases as now also the cache lines of the input may be replaced before all tuples are processed. Thus, each input cache line has to be loaded more than once. The first two terms of $M_{T L B, c}$ are made up analogously. Additionally, using a similar schema as $M_{L i, c}$, the third term models-for relations that contain more pages than there are TLB entries-the additional TLB misses that occur when the number of clusters either approaches the number of available L2 lines $\left(H_{p} \leq|L 2|_{L 2}\right)$ or even exceeds this.

Fig. 14 compares our model (lines) with the experimental results (points) on the Origin2000 for different cardinalities. The model proves to be very accurate for the number of cache misses (both L1 and L2) and TLB misses. The predicted elapsed time is also reasonably accurate on all architectures (cf. Fig. 15). The plots clearly reflect the

5. Using $\min \left\{H_{p},|R e|_{L i}\right\}$ instead of simply $H_{p}$ takes into account that smaller relations may completely fit in $\mathrm{L} i$, i.e., with $H_{p}>|L i|_{L i}>|R e|_{L i}$, several (tiny) clusters share one cache line. 


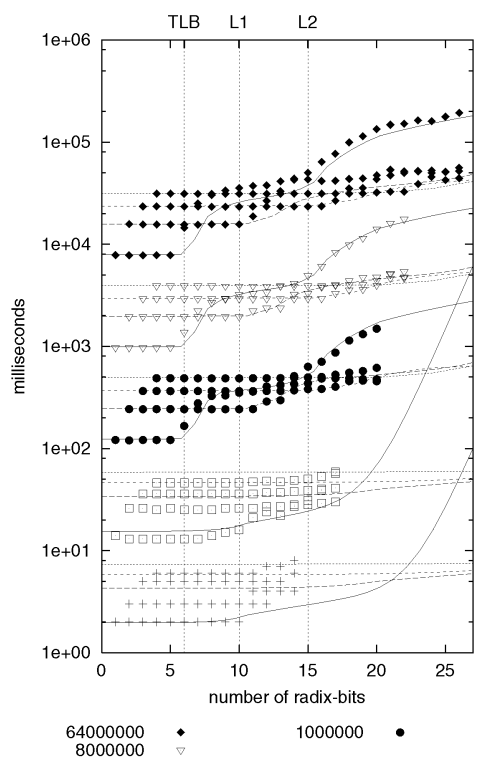

(a)

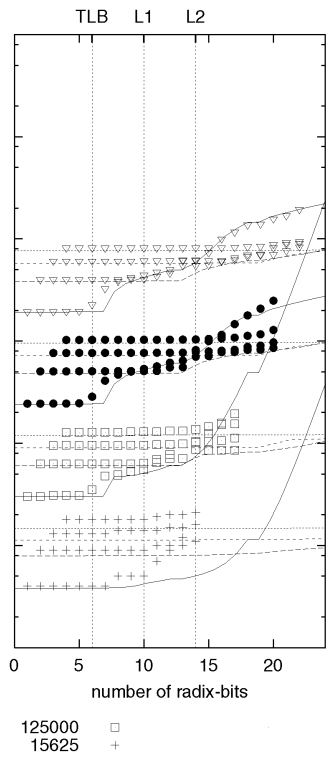

(b)

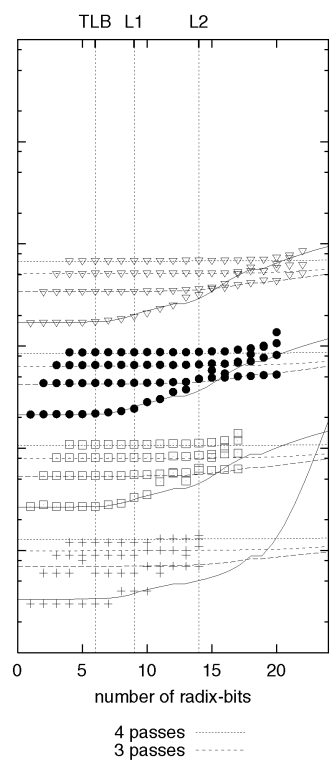

(c)

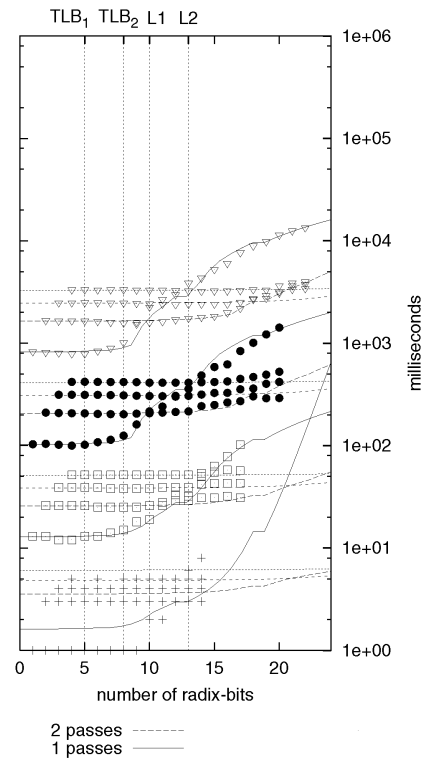

(d)

Fig. 15. Measured (points) and modeled (lines) performance of radix-cluster. (a) Origin2000, (b) Sun Ultra, (c) Intel PC, and (d) AMD PC.

increase in cache and TLB misses and their impact on the execution time whenever the number of clusters per pass exceeds the respective limits.

The question remaining is how to distribute the number of radix-bits over the passes. We conducted another number of experiments using a fixed number of passes, but varying the number of radix-bits per pass. The results showed that even distribution of radix-bits (i.e., $B_{i} \approx \frac{B}{P}, i \in\{1,, P\}$ ) achieves the best performance.

\subsubsection{Isolated Join Performance}

We now analyze the impact of the number of radix-bits on the pure join performance, not including the clustering costs. With 0 radix-bits, the join algorithm behaves like a simple nonpartitioned hash-join.

The partitioned hash-join exhibits increased performance with an increasing number of radix-bits. Fig. 16 shows that this behavior is mainly caused by the memory costs. While the CPU costs are almost independent of the number of radix-bits, the memory costs decreases with an increasing number of radix-bits. The performance increase flattens past the point where the entire inner cluster (including its hash table) consists of fewer pages than there are TLB entries (64). Then, it also fits the L2 cache comfortably. Thereafter, performance increases only slightly until the point that the inner cluster fits the L1 cache. Here, performance reaches its maximum. The fixed overhead by allocation of the hash-table structure causes performance to decrease when the cluster sizes get too small and clusters get very numerous. Again, the Pentium III shows a slightly different behavior. TLB costs do not play any role, but "partial stalls" (i.e., stalls due to dependencies among instructions) are significant with small numbers of radixbits. With an increasing numbers of clusters, the partial stalls decrease, but, then, resource stalls increase, almost neutralizing the memory optimization.

As with radix-cluster, once the memory access is optimized, the execution of partitioned hash-join is dominated by CPU costs. Hence, we applied the same optimizations as above. We inlined the hash-function calls during hash build and hash probe as well as the compare-function call during hash probe and replaced two memcpy by simple assignments, saving five function calls per iteration. Further, we replaced the modulo division ("\%") for calculating the hash index by a bit operation ("\&"). Fig. 17 depicts the original implementation of our hash-join routine and the optimizations we applied.

Fig. 18 shows the execution time breakdown for the optimized partitioned hash-join. For the same reasons as with radix-cluster, the CPU costs are reduced by almost a factor of 4 on the Origin and the Sun, by a factor of 3 on the Pentium III, and by a factor of 2 on the Athlon. The expensive divisions have vanished completely. Additionally, the dependency stalls on the Pentium III have disappeared, but the functional unit stalls remain almost unchanged, now taking about half of the execution time. It is interesting to note the $450 \mathrm{MHz}$ PC outperforms the 250 $\mathrm{MHz}$ Origin on nonoptimized code, but, on CPU optimized code, where both RISC chips execute without any overhead, the PC actually becomes slower due to this phenomenon of resource stalls.

As for the radix-cluster, we also provide a cost model for the partitioned hash-join. The model takes the number of radix-bits, the cardinality, ${ }^{6}$ and the (average) join hit rate as input.

6. For simplicity of presentation, we assume the cardinalities of both input relations to be equal. 


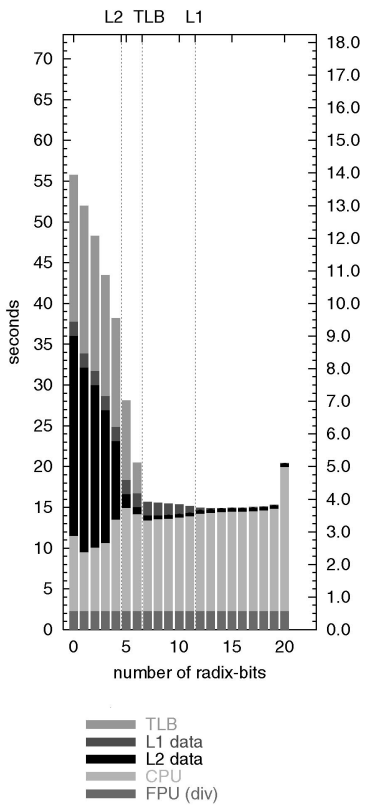

(a)

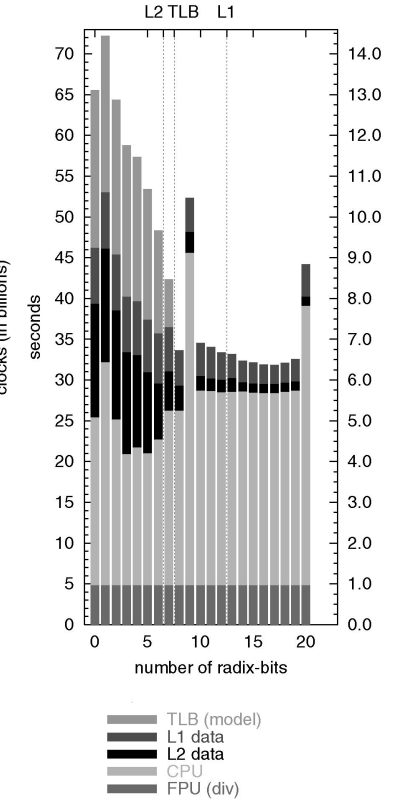

(b)

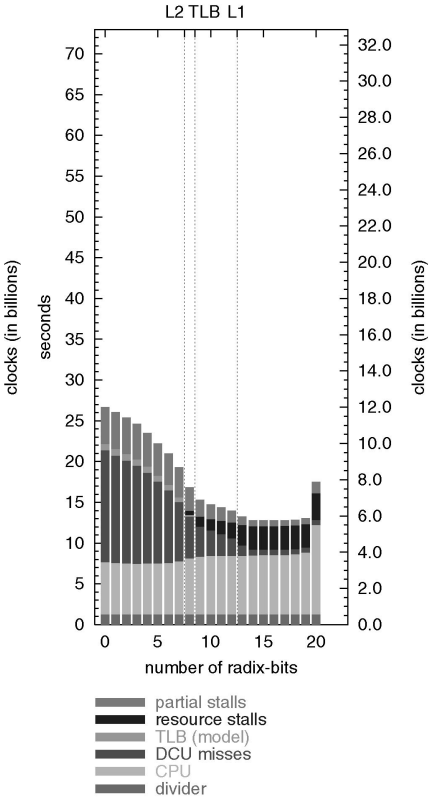

(c)

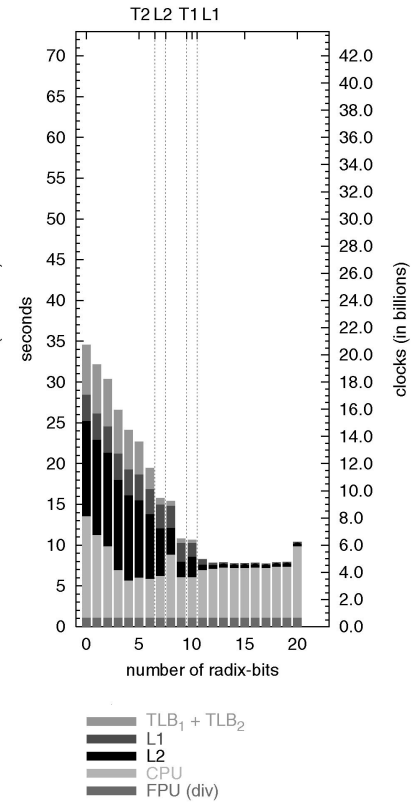

(d)

Fig. 16. Execution time breakdown of partitioned hash-join (cardinality = 8M). (a) Origin2000, (b) Sun Ultra, (c) Intel PC, and (d) AMD PC.

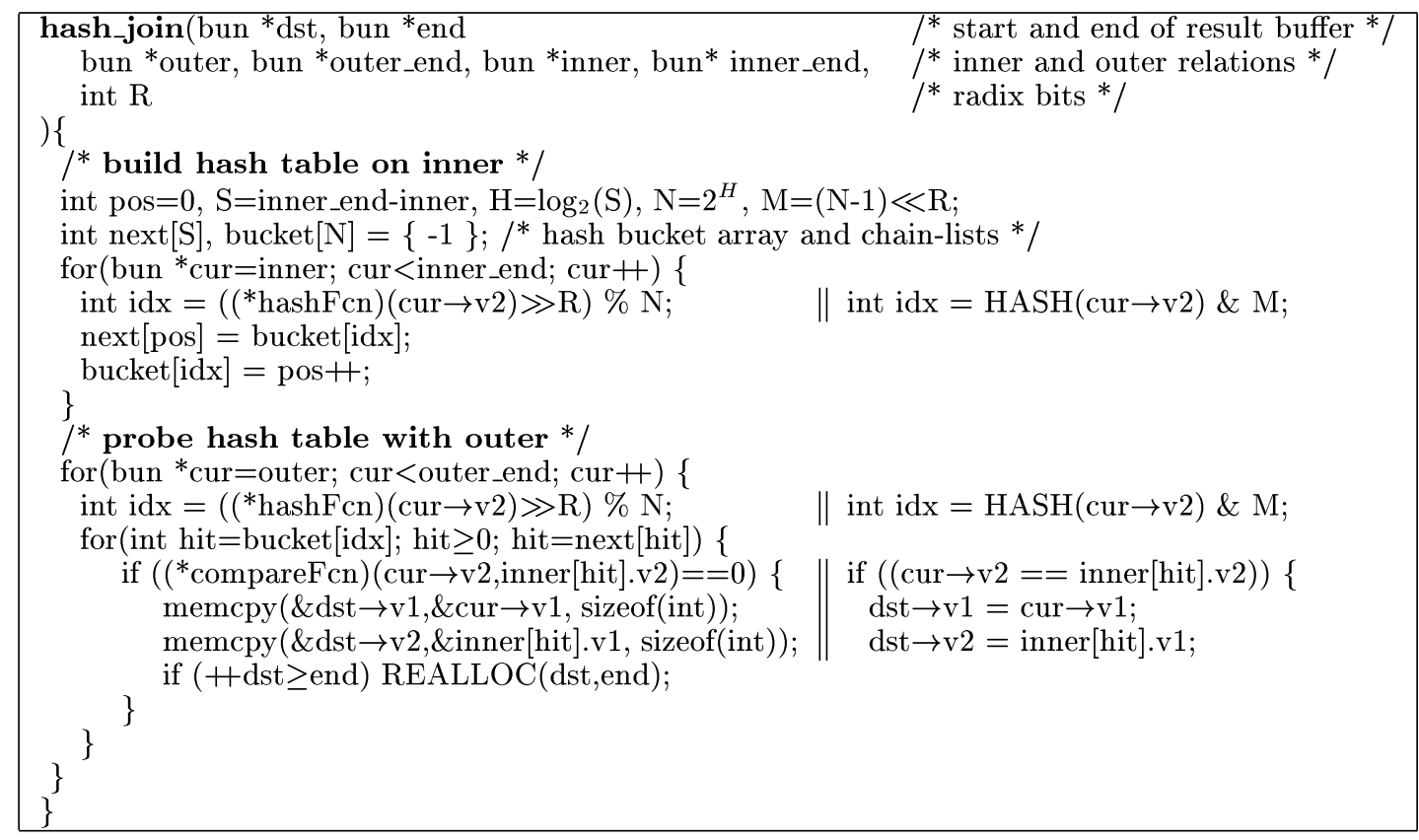

Fig. 17. C language hash-join with annotated CPU optimizations (right).

$$
\begin{aligned}
T_{h}(B, C, r)=C * w_{h} & +M_{L 1, h}(B, C, r) * l_{L 2} \\
& +M_{L 2, h}(B, C, r) * l_{M e m} \\
& +M_{T L B, h}(B, C, r) * l_{T L B} .
\end{aligned}
$$

with

$$
\begin{aligned}
& M_{L i, h}(B, C, r)= \\
& (2+r) *|R e|_{L i}+\left\{\begin{array}{l}
C * \frac{\|C l\|}{\|L i\|}, \\
\text { if }\|C l\| \leq\|L i\| \\
C *(4+2 r) *\left(1-\frac{\|L i\|}{\|C l\|}\right) \\
\text { else }
\end{array}\right.
\end{aligned}
$$

and

$$
\begin{aligned}
& M_{T L B, h}(B, C, r)= \\
& (2+r) *|R e|_{P g}+\left\{\begin{array}{l}
C * \frac{\|C l\|}{\|T L B\|}, \\
\text { if }\|C l\| \leq\|T L B\| \\
C *(4+2 r) *\left(1-\frac{\|T L B\|}{\|C l\|}\right), \\
\text { else. }
\end{array}\right.
\end{aligned}
$$

$|R e|_{L i},|R e|_{P g}$, and $|T L B|$ are as above. $\|C l\|,\|L i\|$, and $\|T L B\|$ denote (in bytes) the cluster size, the sizes of both caches $(i \in\{1,2\})$, and the memory range covered by $|T L B|$ pages, respectively. 


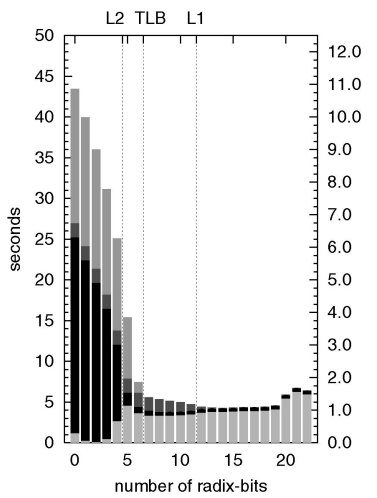

(a)

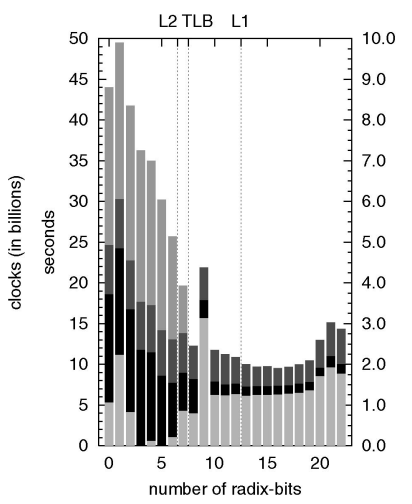

(b)

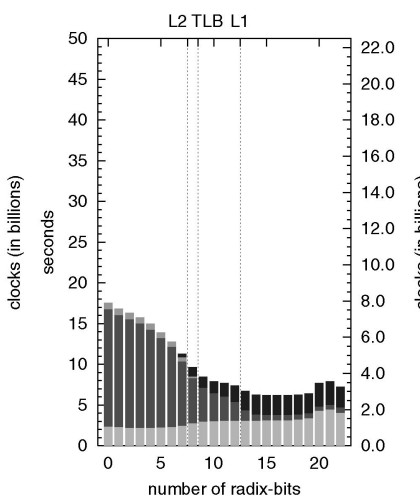

(c)

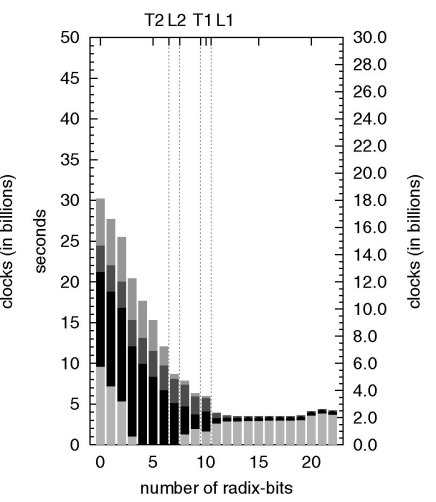

(d)

Fig. 18. Execution time breakdown of optimized partitioned hash-join $(C=8 \mathrm{M})$. (a) Origin2000, (b) Sun Ultra, (3) Intel PC, and (d) AMD PC.

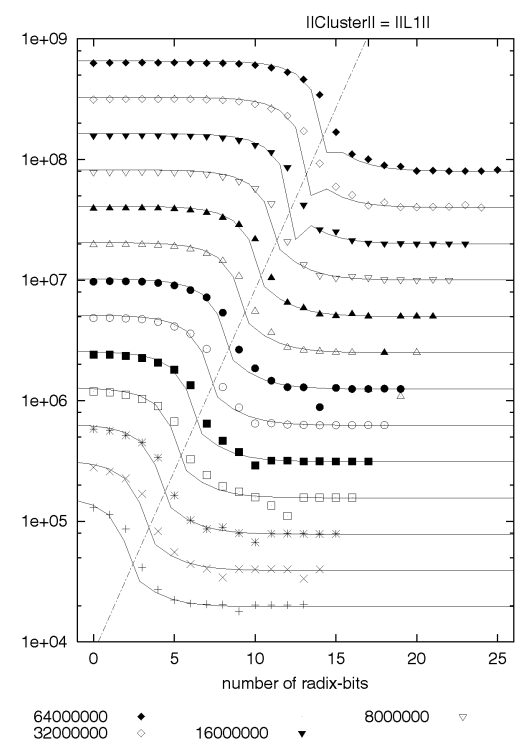

(a)

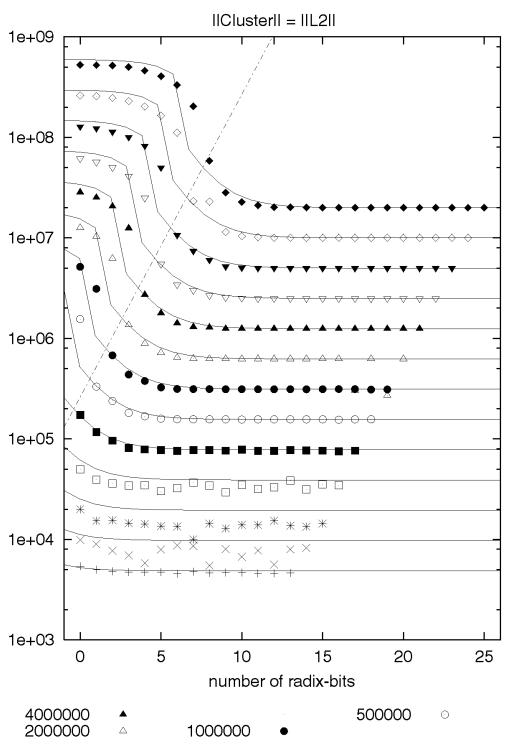

(b)

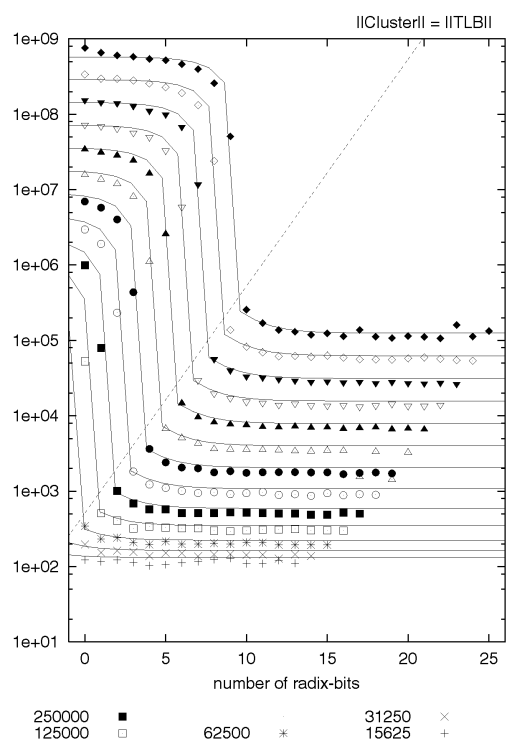

(c)

Fig. 19. Measured (points) and modeled (lines) events of partitioned hash-join (Origin2000). (a) L1 misses, (b) L2 misses, and (c) TLB misses.

$w_{h}$ represents the pure CPU costs per tuple for building the hash-table, doing the hash lookup, and creating the result. We calibrated $w_{h}=440 \mathrm{~ns}$ on the Origin2000, $w_{h}=$ $1100 \mathrm{~ns}$ on the Sun, $w_{h}=711$ ns on the Pentium III (including resource stalls), and $w_{h}=367 \mathrm{~ns}$ on the Athlon.

The first term of $M_{L i, h}$ equals the minimal number of $\mathrm{L} i$ misses for fetching both operands and storing the result. The second term counts the number of additional L $i$ misses when the cluster size either approaches $\mathrm{L} i$ size or even exceeds this. As soon as the clusters get significantly larger than $\mathrm{L} i$, each memory access yields a cache miss due to cache thrashing: four memory accesses per tuple for accessing the outer relation and the bucket array during hash build and hash probe and two memory accesses per join hit to access the inner relation and the chain-lists. The number of TLB misses is modeled analogously.

Figs. 19 and 20 confirm the accuracy of our model (lines) for the number of L1, L2, and TLB misses on the Origin2000, and for the elapsed time on all architectures.

\subsubsection{Overall Join Performance}

After having analyzed the impact of the tuning parameters on the clustering phase and the joining phase separately, we now turn our attention to the combined cluster and join costs. Radix-cluster gets cheaper for fewer radix-bits, whereas partitioned hash-join gets more expensive. Putting together the experimental data we obtained on both clusterand join-performance, we determined the optimum number of $B$ for relation cardinality.

It turns out that there are three possible strategies, which correspond to the diagonals in Fig. 20:

phash L2 partitioned hash-join on $B=\log _{2}(C * 12 /|| L 2||)$ clustered bits, so the inner relation plus hash-table fits the L2 cache. This strategy was used in the work of Shatdal etal. [12] in their partitioned hash-join experiments.

phash TLB partitioned hash-join on $B=\log _{2}(C * 12 /\|T L B\|)$ clustered bits, so the inner relation plus hash-table spans at most $|T L B|$ pages. Our experiments show a significant improvement in the pure join performance between phash L2 and phash TLB. 


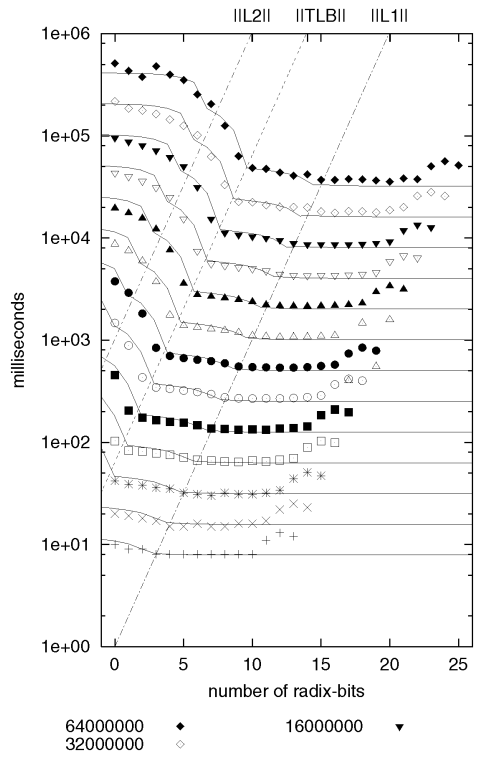

(a)

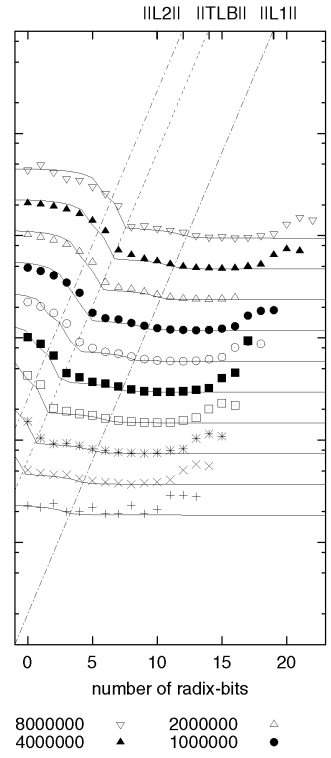

(b)

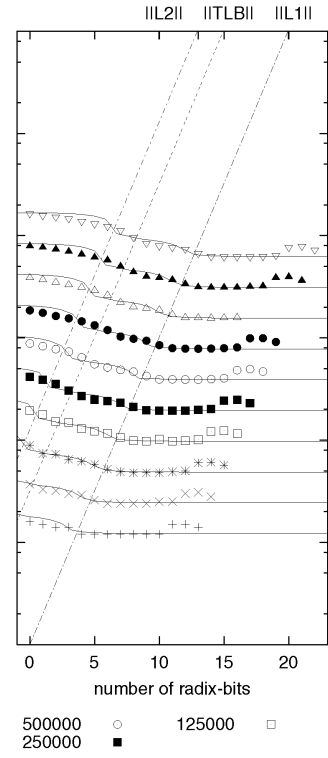

(c)

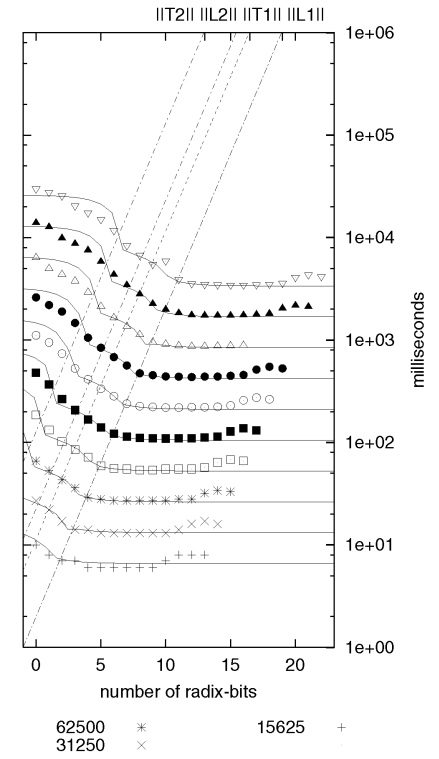

(d)

Fig. 20. Measured (points) and modeled (lines) performance of partitioned hash-join. (a) Origin2000, (b) Sun Ultra, (c) Intel PC, and (d) AMD PC.

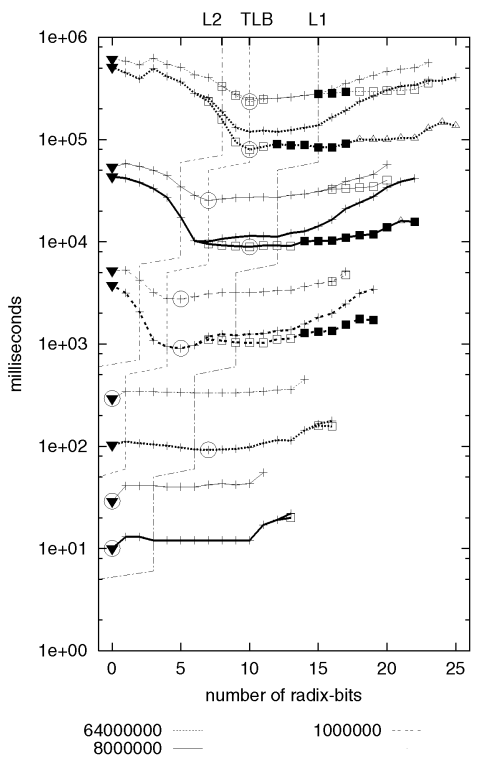

(a)

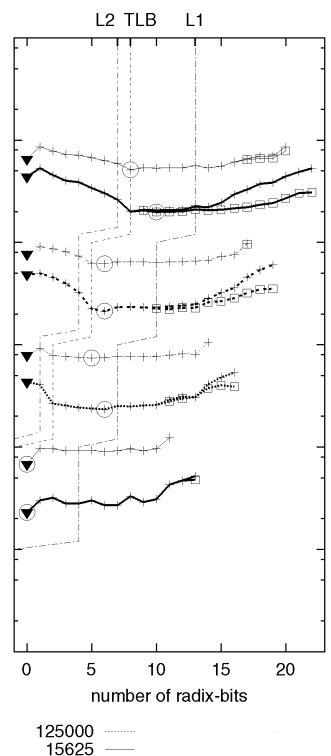

(b)

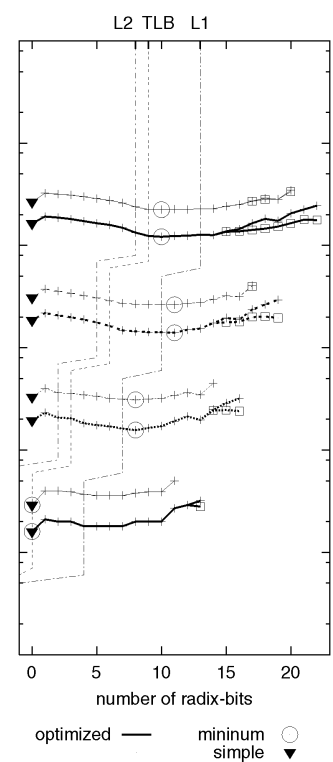

(c)

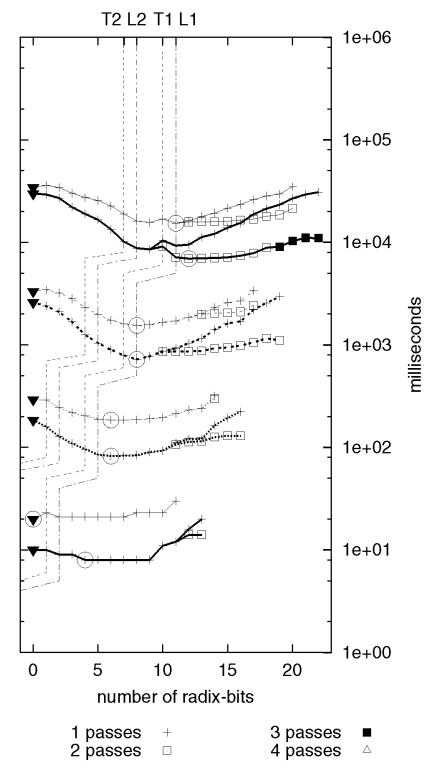

(d)

Fig. 21. Overall performance: nonoptimized (thin lines) vs. optimized (thick lines) implementation. (a) Origin2000, (b) Sun Ultra, (b) Intel PC, and (d) AMD PC.

phash L1 partitioned hash-join on $B=\log _{2}(C * 12 /\|L 1\|)$ clustered bits, so the inner relation plus hash-table fits the L1 cache. This algorithm uses more clustered bits than the previous ones, hence, it really needs the multipass radix-cluster algorithm (a straightforward 1-pass cluster would cause cache thrashing on this many clusters).

Fig. 21 shows the overall performance for the original (thin lines) and the CPU-optimized (thick lines) versions of our algorithms using 1-pass and multipass clustering. In most cases, phash TLB is the best strategy, performing significantly better than phash L2. On the Origin2000 and the Sun, the differences between phash TLB and phash L1 are negligible. On the PCs, phash L1 performs sightly better than phash TLB. With very small cardinalities, i.e., when the relations do not span more memory pages than there are TLB entries, clustering is not necessary and the nonpartitioned hash-join ("simple hash") performs best.

Further, these results show that CPU and memory optimization support each other and boost their effects. The gain of CPU optimization for phash TLB is bigger than that for simple hash and the gain of memory optimization for the CPU-optimized implementation is bigger than that for the nonoptimized implementation. For example, for large relations on the Origin 2000, CPU optimization improves the execution time of simple hash 
by approximately a factor of 1.25 , whereas it yields a factor of 3 with phash TLB. Analogously, memory optimization achieves an improvement of slightly less than a factor of 2.5 for the original implementation, but more than a factor of 5 for the optimized implementation. Combining both optimizations improves the execution time by almost a factor of 10 .

There are two reasons for the boosting effect to occur. First, modern CPUs try to overlap memory access with other useful CPU computations by allowing independent instructions to continue execution while other instructions wait for memory. In a memory-bound load, much CPU computation is overlapped with memory access time, hence, optimizing these computations has no overall performance effect (while it does when the memory access would be eliminated by memory optimizations). Second, an algorithm that allows memory access to be traded for more CPU processing (like radix-cluster) can actually trade more CPU for memory when CPU-cost are reduced, reducing the impact of memory access costs even more.

The Sun Ultra and the AMD PC achieve similar results as the Origin2000, although the absolute gains are somewhat smaller. With the Ultra, the CPU is so slow that trading memory for CPU is less beneficial on this platform; with the AMD PC, the memory access costs are somewhat lower than on the Origin2000, thus offering less potential for improvements.

The overall effect of our optimizations on the Pentium III is just over a factor of 2 . One cause of this is the low memory latency on the Intel PC that limits the gains when memory access is optimized. The second cause is the appearance of the "resource-stalls" which surge in situations where all other stalls are eliminated (and both RISC architectures are really steaming). We expect, though, that future PC hardware with highly parallel IA-64 processors and new Rambus memory systems (which offer high bandwidth but high latencies) will show a more RISC-like performance on our algorithms.

\section{Evaluation}

In the previous section, we demonstrated that performance of large equi-joins can be strongly improved by combining techniques that optimize memory access and CPU resource usage. As discussed in Section 2.6, hardware trends indicate that the effects of such optimizations will become even larger in the future as the memory access bottleneck will deepen and future CPUs will have even more parallel resources. In the following, we discuss the more general implications of these findings to the field of database architecture.

\subsection{Implications for Data Structures}

In terms of data structures for query processing, we already noted from the simple scan experiment in Fig. 4 that full vertical table fragmentation optimizes column wise memory access to table data. This is particularly beneficial if the table is accessed in a sequential scan that reads a minority of all columns. Such table scans very often occur in both OLAP and Data Mining workloads. When record-oriented (i.e., nonfragmented) physical storage is used, such an access leads to data of the nonused columns being loaded into the cache lines, wasting memory bandwidth. In the case of a vertically fragmented table, the table scan just needs to load

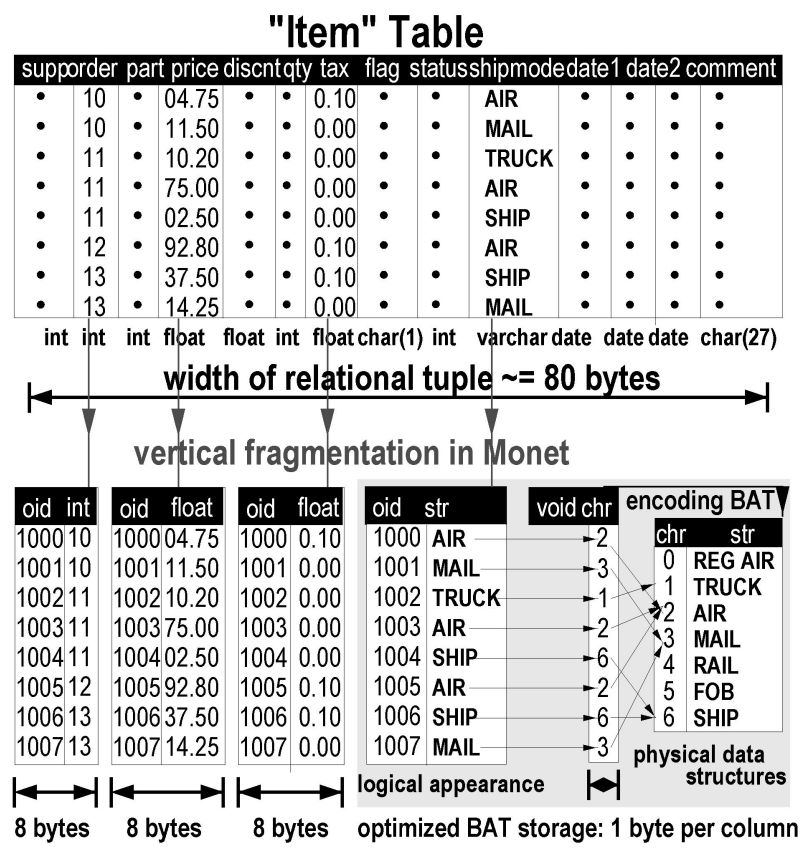

Fig. 22. Vertical decomposition in BATs.

the vertical fragments pertaining to the columns of interest. Reading those vertical fragments sequentially achieves a 100 percent hit rate on all cache levels, exploiting optimal bandwidth on any hardware, including parallel memory access.

There are various ways to incorporate vertical fragmentation in database technology. In Monet, which we designed for OLAP and Data Mining workloads, vertical fragmentation is the basic building block of all physical storage as Monet fully fragments all relations into Binary Association Tables (BATs) (see Fig. 22). Flat binary tables are a simple set-oriented physical representation that is not tied to a particular logical data model, yet is sufficiently powerful to represent, e.g., join indices [35]. Monet has successfully been used to store and query relational, object-oriented, and network data structures using this very simple data model and a small kernel of algebraic operations on it [8]. In Monet, we applied two additional optimizations that further reduce the per-tuple memory requirements in its BATs:

- Virtual-OIDs. Generally, when decomposing a relational table, we get an identical system-generated column of OIDs in all decomposition BATs, which is dense and ascending (e.g., 1,000, 1001, .., 1007). In such BATs, Monet computes the OID-values on-thefly when they are accessed, using positional lookup of the BUN, and avoids allocating the 4-byte OID field. This is called a "virtual-OID" or VOID column. Apart from reducing memory requirements by half, this optimization is also beneficial when joins or semijoins are performed on OID columns. ${ }^{7}$ When one of the join columns is VOID, Monet uses

7. In Monet, the projection phase in query processing typically leads to additional "tuple-reconstruction" joins on OID columns that are caused by the fact that tuples are decomposed into multiple BATs. 
positional lookup instead of, e.g., hash-lookup, effectively eliminating all join costs.

- Byte-encodings. Database columns often have a low domain cardinality. For such columns, Monet uses fixed-size encodings in 1 or 2-byte integer values. This simple technique was chosen because it does not require decoding effort when the values are used (e.g., a selection on a string "MAIL" can be remapped to a selection on a byte with value of 3). A more complex scheme (e.g., using bit-compression) might yield even more memory savings, but the decoding-step required whenever values are accessed can quickly become counterproductive due to extra CPU effort. Even if decoding would just cost a handful of cycles per tuple, this would more than double the amount of CPU effort in simple database operations like a simple aggregation from Section 2.2, which takes just two cycles of CPU work per tuple.

Fig. 22 shows that, when applying both techniques, the storage needed for 1 BUN in the "shipmode" column is reduced from 8 bytes to just one. Reducing the stride from 8 to 1 byte significantly enhances performance in the scan experiment from Fig. 4, eliminating all memory access costs.

Alternative ways of using vertical table fragmentation in a database system are to offer the logical abstraction of relational tables but employ physically fragmentation in transposed files [36] on the physical level (as in NonStopSQL [37]) or to use vertically fragmented data as a search accelerator structure similar to a B-tree. Sybase IQ uses this approach as it automatically creates projection indices on each table column [20]. In the end, however, all these approaches lead to the same kind and degree of fragmentation.

\subsection{Implications for Implementation Techniques}

Implementation techniques strongly determine how CPU and memory are used in query processing and have been the subject of study in the field of main-memory database engineering [23], where query processing costs are dominated by CPU processing. First, we present some rules of thumb that specifically take into account the modern hardware optimization aspects, then we explain how they were implemented in Monet:

- Use the most efficient algorithm. Even the most efficient implementation will not make a suboptimal algorithm perform well. A more subtle issue is tuning algorithms with the optimal parameters.

- Minimize memory copying. Buffer copying should be minimized as it both wastes CPU cycles and also causes spurious main-memory access. As function calls copy their parameters on the stack, they are also a source of memory copying and should be avoided in the innermost loops that iterate over all tuples. A typical function call overhead is about $20 \mathrm{CPU}$ cycles.

- Allow compiler optimizations. Techniques like memory prefetching and generation of parallel EPIC code in the IA-64 rely on compilers to detect the independence of certain statements. These compiler optimizations work especially well if the hotspot of the algorithm is one simple loop that is easily analyzable for the compiler. Again, performing function calls in these loops forces the compiler to assume the worst (side effects) and prevent optimizations from taking place. This especially holds in database code, where those function calls cannot be analyzed at compile time, since the database atomic type interface makes use of $\mathrm{C}$ dereferenced calls on a function-pointer looked up in an ADT table or $\mathrm{C}++$ late-binding methods.

As an example of correctly tuning algorithms, we discuss the (nonpartitioned) hash-join implementation of Monet that uses a simple bucket-chained hash-table. In a past implementation, it used a default mean bucket chain length of four [38], where, actually, a length of one is optimal (perfect hashing). Also, we had used integer division (modulo) by a prime-number (the number of hash buckets) to obtain a hash-bucket number, while integer division costs 40-80 cycles on current CPUs. Later, we changed the number of hash buckets to be a power of 2 (i.e., $N=2^{x}$ ) and, hence, we could replace the expensive modulo division by a much cheaper bit-wise AND with $N-1$. Such simple tuning made the algorithm more than four times faster.

In order to minimize copying, Monet does not do explicit buffer management, rather it uses virtual memory to leave this to the OS. This avoids having to copy tuple segments in and out of a buffer manager whenever the DBMS accesses data. Monet maps large relations stored in a file into virtual memory and accesses it directly. Minimizing memory copying also means that pointer swizzling is avoided at all times by not having hard pointers and value-packing in any data representation.

Functions calls are minimized in Monet by applying logarithmic code expansion [7]. Performance-critical pieces of code, like the hash-join implementation, are replicated in specific functions for the most commonly used types. For example, the hash-join is separated in an integer-join, a string-join, and an ADT join, etc. (that handles all other types). The specific integer-join processes the table values directly as $C$ integers without calling a hash-function for hashing or calling a comparison function when comparing two values. The same technique is applied for constructing the result relation, eliminating function calls for inserting the matching values in the result relation. To make this possible, the type-optimized join implementations require the result to have a fixed format: a join index containing OIDs (in Monet, the result of joining two BATs is again a BAT, so it has a fixed binary format and typical invocations produce a BAT with matching OID pairs). In this way, all function calls can be removed from an algorithm in the optimized cases. For the nonoptimized cases, the (slower) but equivalent implementation is employed that uses ADT method calls for manipulating values. The Monet source code is kept small by generating both the optimized and ADT code instantiations with a macropackage from one template algorithm. We refer to [8] for a detailed discussion of this subject.

\subsection{Implications for Query Processing Algorithms}

Our join experiments demonstrated that performance can strongly improve when algorithms that have a random memory access pattern are tuned in order to ensure that the randomly accessed region does not exceed the cache size (be it L1, L2, or TLB). In the case of join, we confirmed the 
results of Shatdal et al., who had proposed a partitioned hash-join such that each partition joined fits the L2 cache [12] and showed that the beneficial effect of this algorithm is even stronger on modern hardware. Second, we introduced a new partitioning algorithm, called radix-cluster, that performs multiple passes over the data to be partitioned but earns back this extra CPU work with much less memory access cost when the number of partitions gets large.

We believe that similar approaches can be used to optimize algorithms other than equi-join. For instance, Ronström [39] states that a B-tree with a block-size equal to the L2 cache line size as a main-memory search accelerator now outperforms the traditionally best-known main-memory T-tree search structure [13]. As another example, memory cost optimizations can be applied to sorting algorithms (e.g., radix-cluster followed by quicksort on the partitions) and might well change the trade-offs for other well-known main-memory algorithms (e.g., radix-sort has a highly cachable memory access pattern and is likely to outperform quicksort).

Main-memory cost models are a prerequisite for tuning the behavior of an algorithm to optimize memory cache usage as they allow us to make good optimization decisions. Our work shows that such models can be obtained and how to do it. First, we show, with our calibration tool, how all relevant hardware characteristics can be retrieved from a computer system automatically. This calibrator does not need any OS support whatsoever and should, in our opinion, be used in modern DBMS query optimizers. Second, we present a methodological framework that first characterizes the memory access pattern of an algorithm to be modeled in a formula that counts certain hardware events. These computed events are then scored with the calibrated hardware parameters to obtain a fullcost model. This methodology represents an important improvement over previous work on main-memory cost models [24], [25], where performance is characterized on the coarse level of a procedure call with "magical" cost factors obtained by profiling. We were helped in formulating this methodology through our usage of hardware event counters present in modern CPUs.

We think our findings are not only relevant to mainmemory databases engineers. Vertical fragmentation and memory access costs have a strong impact on performance of database systems at a macrolevel, including those that manage disk-resident data. Nyberg et al. [40] stated that techniques like software-assisted disk-striping have reduced the I/O bottleneck, i.e., queries that analyze large relations (like in OLAP or Data Mining) now read their data faster than it can be processed. Hence, the main performance bottleneck for such applications is shifting from $\mathrm{I} / \mathrm{O}$ to memory access. We therefore think that, as the I/O bottleneck decreases and the memory access bottleneck increases, main-memory optimization of both data structures and algorithms-as described in this paper-will become a prerequisite to any DBMS for exploiting the power of custom hardware.

In Monet, we delegate $\mathrm{I} / \mathrm{O}$ buffering to the OS by mapping large data files into virtual memory, hence treating management of disk-resident data as memory with a large granularity (a memory page is like a large cache line). This is in line with the consideration that disk-resident data is the bottom level of a memory hierarchy that goes up from the virtual memory to the main memory through the cache memories up to the CPU registers (Fig. 3). Algorithms that are tuned to run well on one level of the memory also exhibit good performance on the lower levels.

\section{ConClusion}

We have shown what steps are taken in order to optimize the performance of large main-memory joins on modern hardware. To achieve better usage of scarce memory bandwidth, we recommend using vertically fragmented data structures. We refined partitioned hash-join with a new partitioning algorithm, called radix-cluster, which prevents performance becoming dominated by memory latency (avoiding the memory access bottleneck). Exhaustive equi-join experiments were conducted on modern SGI, Sun, Intel, and AMD hardware. We formulated detailed analytical cost models that explain why this algorithm makes optimal use of hierarchical memory systems found in modern computer hardware and very accurately predicted performance on all three platforms. Further, we showed that, once memory access is optimized, CPU resource usage becomes crucial for the performance. We demonstrated, how CPU resource usage can be improved by using appropriate implementation techniques. The overall speedup obtained by our techniques can be almost an order of magnitude. Finally, we discussed the consequences of our results in a broader context of database architecture and made recommendations for future systems.

\section{References}

[1] T.C. Mowry, "Tolerating Latency Through Software-Controlled Data Prefetching," PhD thesis, Computer Science Dept., Stanford Univ., 1994.

[2] A.G. Ailamaki, D.J. DeWitt, M.D. Hill, and D.A. Wood, "DBMSs on a Modern Processor: Where Does Time Go?" Proc. Int'l Conf. Very Large Data Bases (VLDB), pp. 266-277, Sept. 1999.

[3] L.A. Barroso, K. Gharachorloo, and E.D. Bugnion, "Memory System Characterization of Commercial Workloads," Proc. Int'l Symp. Computer Architecture, June 1998.

[4] K. Keeton, D.A. Patterson, Y.Q. He, R.C. Raphael, and W.E. Baker, "Performance Characterization of a Quad Pentium Pro SMP Using OLTP Workloads," Proc. Int'l Symp. Computer Architecture, pp. 1526, June 1998.

[5] P. Trancoso, J.L. Larriba-Pey, Z. Zhang, and J. Torellas, "The Memory Performance of DSS Commercial Workloads in SharedMemory Multiprocessors," Proc. Int'l Symp. High Performance Computer Architecture, Jan. 1997.

[6] Silicon Graphics, Inc., Performance Tuning and Optimization for Origin2000 and Onyx2. Jan. 1997.

[7] M. Kersten, "Using Logarithmic Code-Expansion to Speedup Index Access and Maintenance," Proc. Int'l Conf. Foundation on Data Organization and Algorithms, pp. 228-232, Oct. 1989.

[8] P. Boncz and M. Kersten, "MIL Primitives For Querying a Fragmented World," The VLDB J., vol. 8, no. 2, pp. 101-119, Oct. 1999.

[9] P.M.G. Apers, C.A. van den Berg, J. Flokstra, P.W.P. J. Grefen, M. Kersten, and A.N. Wilschut, "PRISMA/DB: A Parallel Main Memory Relational DBMS," IEEE Trans. Knowledge and Data Eng., vol. 4, no. 6, pp. 541-554, Dec. 1992.

[10] P. Boncz, W. Quak, and M. Kersten, "Monet and Its Geographical Extensions: A Novel Approach to High-Performance GIS Processing," Proc. Int'l Conf. Extending Database Technology, pp. 147-166, June 1996.

[11] P. Boncz, A.N. Wilschut, and M. Kersten, "Flattening an Object Algebra to Provide Performance," Proc. IEEE Int'l Conf. Data Eng., pp. 568-577, Feb. 1998. 
[12] A. Shatdal, C. Kant, and J. Naughton, "Cache Conscious Algorithms for Relational Query Processing," Proc. Int'l Conf. Very Large Data Bases (VLDB), pp. 510-512, Sept. 1994.

[13] T.J. Lehman and M.J. Carey, "A Study of Index Structures for Main Memory Database Management Systems," Proc. Int'l Conf. Very Large Data Bases (VLDB), pp. 294-303, Aug. 1986.

[14] T.J. Lehman and M.J. Carey, "Query Processing in Main Memory Database Systems," Proc. ACM SIGMOD Int'l Conf. Management of Data, pp. 239-250, May 1986.

[15] M.H. Eich, "Main Memory Database Research Directions," Proc. Sixth Int'l Workshop Database Machines, pp. 251-268, June 1989.

[16] A. Wilschut, "Parallel Query Execution in a Main-Memory Database System," PhD thesis, Universiteit Twente, 1991.

[17] A. Analyti and S. Pramanik, "Fast Search in Main Memory Databases," Proc. ACM SIGMOD Int'l Conf. Management of Data, pp. 215-224, June 1992.

[18] H. Garcia-Molina and K. Salem, "Main Memory Database Systems: An Overview," IEEE Trans. Knowledge and Data Eng., vol. 4, no. 6, pp. 509-516, Dec. 1992.

[19] Times Ten Team, "In-Memory Data Management for Consumer Transactions the Times-Ten Approach," ACM SIGMOD Record, vol. 28 , no. 2 , pp. 528-529, June 1999.

[20] Sybase Corp., “Adaptive Server IQ,"Whitepaper, July 1996.

[21] Compaq Corp., "Infocharger," Whitepaper, Jan. 1998.

[22] M. Kersten, A.P.J.M. Siebes, M. Holsheimer, and F. Kwakkel, "Research and Business Challenges in Data Mining Technology," Proc. Datenbanken in Büro, Technik und Wissenschaft, pp. 1-16, Mar. 1997.

[23] D.J. DeWitt, R.H. Katz, F. Olken, L.D. Shapiro, M. Stonebraker, and D.A. Wood, "Implementation Techniques for Main Memory Database Systems," Proc. ACM SIGMOD Int'l Conf. Management of Data, pp. 1-8, June 1984.

[24] S. Listgarten and M.-A. Neimat, "Modelling Costs for a MMDBMS," Proc. Int'l Workshop Real-Time Databases, Issues, and Applications, pp. 72-78, Mar. 1996.

[25] K.-Y. Whang and R. Krishnamurthy, "Query Optimization in a Memory-Resident Domain Relational Calculus Database System," ACM Trans. Database Systems, vol. 15, no. 1, pp. 67-95, Mar. 1990.

[26] R. Berrendorf and H. Ziegler, "PCL-The Performance Counter Library," Techical Report FZJ-ZAM-IB-9816, ZAM, Forschungzentrum Jülich, Germany, 1998

[27] M. Zagha, B. Larson, S. Turner, and M. Itzkowitz, "Performance Analysis Using the MIPS R10000 Performance Counters," Proc. Supercomputing '96 Conf., Nov. 1996.

[28] K. Yeager, "The MIPS R10000 Superscalar Microprocessor," IEEE Micro, vol. 16, no. 2, pp. 28-40, Apr. 1996.

[29] G.P. Copeland and S. Khoshafian, "A Decomposition Storage Model," Proc. ACM SIGMOD Int'l Conf. Management of Data, pp. 268-279, May 1985.

[30] Rambus Technologies, Inc., Direct Rambus Technology Disclosure, 1996, www.rambus.com/docs/drtechov.pdf.

[31] D. Patterson, T. Anderson, N. Cardwell, R. Fromm, K. Keeton, C. Kozyrakis, R. Thomas, and K. Yelick, "A Case for Intelligent RAM," IEEE Micro, vol. 17, no. 2, pp. 34-44, Mar. 1997.

[32] S. McKee, R. Klenke, K. Wright, W. Wulf, M. Salinas, J. Aylor, and A. Batson, "Smarter Memory: Improving Bandwidth for Streamed References," Computer, vol. 31, no. 7, pp. 54-63, July 1998.

[33] Sematech, National Roadmap For Semiconductor Technology: Technology Needs, 1997, http://www.itrs.net/ntrs/publntrs.nsf.

[34] D. August, D. Connors, S. Mahlke, J. Sias, K. Crozier, B. Cheng, P. Eaton, Q. Olaniran, and W. Hwu, "Integrated Predicated and Speculative Execution in the IMPACT EPIC Architecture," Proc. Int'l Symp. Computer Architecture, pp. 227-237, June 1998.

[35] P. Valduriez, "Join Indices," ACM Trans. Database Systems, vol. 12, no. 2, pp. 218-246, June 1987.

[36] D.S. Batory, "On Searching Transposed Files," ACM Trans. Database Systems, vol. 4, no. 4, pp. 531-544, 1979.

[37] J. Clear, D. Dunn, B. Harvey, M. Heytens, P. Lohman, A. Mehta, M. Melton, H. Richardson, L. Rohrberg, A. Savasere, R. Wehrmeister, and M. Xu, "NonStopSQL/MX," Proc. Int'l Conf. Knowledge Discovery and Data Mining, Aug. 1999.

[38] P. Boncz, S. Manegold, and M. Kersten, "Database Architecture Optimized for the New Bottleneck: Memory Access," Proc. Int'l Conf. Very Large Data Bases (VLDB), pp. 54-65, Sept. 1999.

[39] M. Ronström, "Design and Modeling of a Parallel Data Server for Telecom Applications," PhD thesis, Linköping Univ., 1998.
[40] C. Nyberg, T. Barclay, Z. Cvetanovic, J. Gray, and D. Lomet, "AlphaSort: A RISC Machine Sort," Proc. ACM SIGMOD Int'l Conf. Management of Data, pp. 233-242, May 1994.

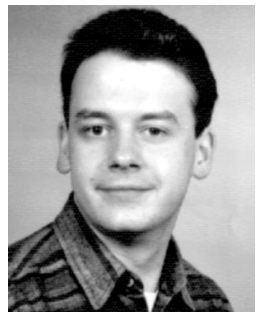

Stefan Manegold received the MSc degree in computer science from the Technical University Clausthal, Germany, in 1994. From 1995 until 1997, he was working as a research assistant with the Database Research Group at the Institute for Informatics of Humboldt University, Berlin, Germany. Since 1997, he has been working as a database researcher with the Database Research Group at the Centrum voor Wiskunde en Informatica (CWI), Amsterdam, The Netherlands. He is about to finish his PhD track, investigating performance issues and cost modeling in main-memory database systems. His research interests include distributed and parallel database systems, main-memory database systems, query processing, and cost models.

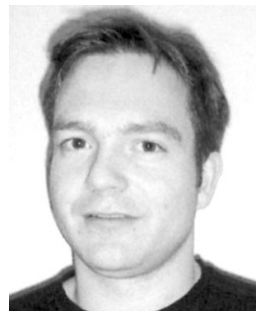

Peter Boncz received the MSc degree in computer science from Vrije Universiteit in 1992 and the $\mathrm{PhD}$ degree in computer science from the University of Amsterdam in 2002. During his $\mathrm{PhD}$ track at the Database Research Group of the CWI with Professor Kersten, he investigated database architecture for queryintensive applications like OLAP and Data Mining. This research led to the development of the Monet database kernel. During the past seven years, he has published a series of articles on various aspects of the Monet system in prominent database journals and conferences. The Monet system is used at various sites in academia for research into multimedia, GIS, XML, and medical database systems, as well as commercially by Data Distilleries, a CWI-spinoff company that creates data mining products. From 1998 until 2001, Dr. Boncz was working at Data Distilleries as chief architect. His research interests include: database architecture, parallel database systems, query languages, query optimization, extensibility in database systems, database kernel design and implementation, and computer architecture. He currently acts as a program committee member of the VLDB Conference. He is a member of the IEEE.

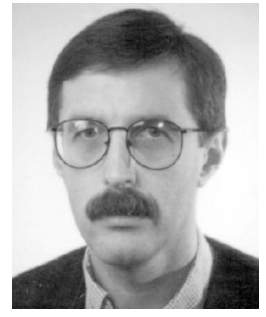

Martin Kersten received the $\mathrm{PhD}$ degree in computer science from Vrije Universiteit in 1985 on research in database security, whereafter he moved to CWI to establish the Database Research Group. In his professional career, he has developed three complete database kernels. From 1979 until 1985, he developed a small relational kernel, called Troll, which was sold as part of a CASE tool, 1985-1991. Between 1986 and 1991, he was codesigner of the PRISMA database machine, a RDBMS for a 100-node multiprocessor based on the assumption that the hotset is memory resident. In 1992, he initiated the development of his third DBMS, called Monet. This system is an extensible main-memory-oriented DBMS which is currently used in a commercial data mining system and the pivot in several national projects aimed at advanced database applications, such as image processing and geographical information systems. Currently, he is heading a department involving 50 researchers in areas covering data mining and data warehousing, multimedia information systems, information engineering, and quantum computing. Since 1994, he has been a professor at the University of Amsterdam. In 1995, he cofounded Data Distilleries. He has published more than 130 scientific papers and is a member of the editorial board of the VLDB Journal and Parallel and Distributed Systems. He acts as a reviewer for ESPRIT projects and is a trustee of the VLDB Endowment board. He is a member of the IEEE Computer Society.

$\triangleright$ For more information on this or any computing topic, please visit our Digital Library at http://computer.org/publications/dlib. 